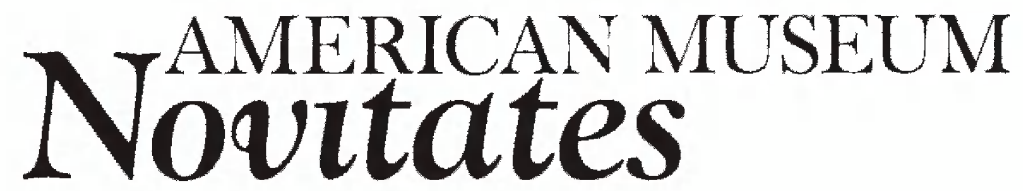

PUBLISHED BY THE AMERICAN MUSEUM OF NATURAL HISTORY CENTRAL PARK WEST AT 79TH STREET, NEW YORK, NY 10024 Number 3596, 30 pp., 106 figures

December 12, 2007

\title{
A Taxonomic Review of the Trapdoor Spider Genus Myrmekiaphila (Araneae, Mygalomorphae, Cyrtaucheniidae)
}

\author{
JASON E. BOND ${ }^{1}$ AND NORMAN I. PLATNICK ${ }^{2}$
}

\begin{abstract}
The mygalomorph spider genus Myrmekiaphila comprises 11 species known only from the southeastern United States. The type species, M. foliata Atkinson, is removed from the synonymy of $M$. fluviatilis (Hentz) and placed as a senior synonym of $M$. atkinsoni Simon. A neotype is designated for M. fluviatilis and males of the species are described for the first time. Aptostichus flavipes Petrunkevitch is transferred to Myrmekiaphila. Six new species are described: M. coreyi and $M$. minuta from Florida, $M$. neilyoungi from Alabama, M. jenkinsi from Tennessee and Kentucky, and M. millerae and $M$. howelli from Mississippi.
\end{abstract}

\section{INTRODUCTION}

The trapdoor spider genus Myrmekiaphila (Cyrtaucheniidae, Euctenizinae) has long remained in relative obscurity. Aside from occasional species descriptions, no significant taxonomic work on the group has appeared. Members of the genus are widely distributed throughout the southeastern United States (fig. 1), ranging from northern Virginia along the Appalachian Mountains southward through West Virginia, Kentucky, North and South Carolina, Tennessee, and northern Georgia into the Southeastern Plains and Southern Coastal Plain of Alabama, Mississippi, and Florida. The range of the genus

\footnotetext{
${ }^{1}$ Research Associate, Division of Invertebrate Zoology, American Museum of Natural History; Associate Professor, Department of Biology, Howell Science Complex N211, East Carolina University, Greenville, NC 27858 (bondja@ ecu.edu).

${ }^{2}$ Peter J. Solomon Family Curator, Division of Invertebrate Zoology, American Museum of Natural History; Adjunct Professor, Department of Biology, City College, City University of New York; Adjunct Professor, Department of Entomology, Cornell University; Adjunct Senior Research Scientist, Center for Environmental Research and Conservation, Columbia University (platnick@amnh.org).
} 


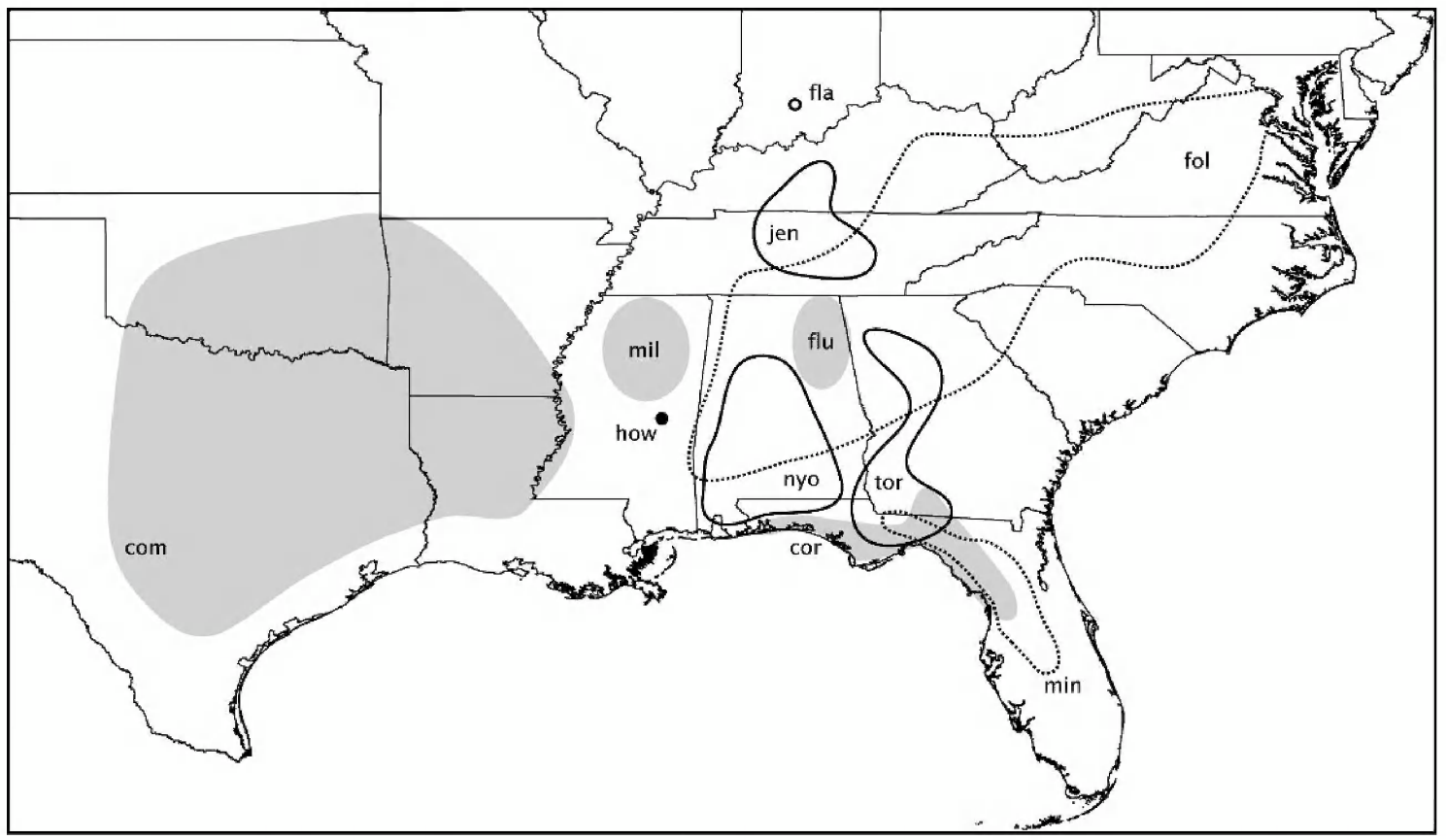

Fig. 1. Generalized distribution map for Myrmekiaphila species. com - M. comstocki, cor - M. coreyi, folM. foliata, fla - M. flavipes, fluv - M. fluviatilis, how - M. howelli, jen - M. jenkinsi, mil - M. millerae, min $M$. minuta, nyo - M. neilyoungi, tor - M. torreya.

also extends west into southern Indiana, Louisiana, Arkansas, Oklahoma, and eastern and central Texas. Thus, Myrmekiaphila species are found in a diverse set of habitats and ecoregions, ranging from the relatively high elevation temperate deciduous forests of the southern Appalachian Mountains to the more xeric, low elevation climates of central Texas.

Members of the genus are homogenous in general morphology and behavior. Females are moderate in size for trapdoor spiders and tend to be uniform in coloration across taxa (figs. 2, 3); most live specimens show some evidence of dusky stripes on the dorsum of the abdomen. Males are easily distinguished from other members of the North American mygalomorph fauna by the modified palpal tibia (figs. 4-13). All members of the genus appear to live in subterranean, silk-lined burrows, covered by a silken-soil trapdoor, from which the spiders forage. Some Myrmekiaphila species appear unique among cyrtaucheniids because they construct burrow side chambers that can be closed off from the main burrow chamber by a second trapdoor (Atkinson
1886, and personal observations). Some other North American euctenizines build burrows with side chambers (e.g., Promyrmekiaphila Schenkel, Aptostichus Simon); however, they do not close the below-ground chambers with trapdoors.

The family level placement of the genus, although recently addressed, remains to be fully resolved. Myrmekiaphila was transferred from the Ctenizidae to the Cyrtaucheniidae, and placed in the subfamily Euctenizinae, by Raven (1985). Recent phylogenetic analyses of the Mygalomorphae (Bond and Hedin, 2006; Hedin and Bond, 2006) show the subfamily Euctenizinae to be monophyletic but indicate that serious reconsideration of the family Cyrtaucheniidae is warranted; Myrmekiaphila will likely be placed in a different family in the near future. Within the North American euctenizine clade, Myrmekiaphila appeared as the sister group to all the southwestern genera save Apomastus Bond and Opell (the sister group to all euctenizines) in the results of Bond and Hedin (2006). This result differed from that reported by Bond and Opell (2002), who placed Myrmekiaphila as the sister group 
to all North American euctenizines plus Apomastus.

As with many mygalomorph groups, the taxonomic history of Myrmekiaphila is relatively brief. The genus was first described by Atkinson (1886) for the type species $M$. foliata. Eventually synonymized with $M$. fluviatilis (Hentz, 1850) by Banks (1900), M. foliata erroneously remained in synonymy through the works of a number of authors (Simon, 1892; Bishop and Crosby, 1926; Petrunkevitch, 1929). Only three additional species have been described: $M$. atkinsoni Simon (1891), M. comstocki Bishop and Crosby (1926), and M. torreya Gertsch and Wallace (1936). Although a number of workers (Roth, 1994; Bond, 2005) recognized the existence of additional species diversity within the genus, we present here the first comprehensive taxonomic review of the group.

\section{MATERIAL, METHODS, AND ABBREVIATIONS}

Unique specimen voucher numbers were assigned to all specimens (MYR001-MYR293 and MYxxxx); a label with the voucher number was added to each vial. All collecting localities were georeferenced using a Global Positioning System receiver or by finding the approximate locality, based on collecting label data, on United States Geological Survey topographic maps or with Google Earth (Google Inc., Mountain View, CA). Latitude and longitude data approximated in the latter manner are provided in brackets [ ] in the material examined sections. Because many of the older collecting labels lack sufficient locality information, many of the georeferenced values are imprecise and should be used with caution. Data for labels that document only county and/or town information were georeferenced to the approximate geographic center of the locality. Detailed locality data and associated GIS data can be downloaded at http://www.mygalomorphae.org as an Excel file and as a Biota (Colwell, 2003) data set.

All measurements are in millimeters and were taken with a Leica MZ 9.5 stereomicroscope equipped with a $10 \times$ ocular and ocular micrometer scale. Measurements, unless otherwise stated, were taken from the left appendage, in retrolateral view, using the highest magnification possible. Lengths of leg articles were taken from the midlineproximal point of articulation to the midlinedistal point of the article. Color images of male palps and leg I were prepared using a Microptics Imaging System (Microptics, Inc., Ashland, VA). Carapace and leg coloration are described using Munsell ${ }^{\circledR}$ Color Charts (Windsor, NY) and are given using the color name and color notation (hue value/chroma). Palpal bulbs examined using scanning electron microscopy were air-dried and sputter coated with gold prior to viewing on an FEI Quanta 200. Spermathecae were dissected from the specimen, cleared in clove oil, and examined under a compound microscope.

The species description format and features assessed follow Bond (2004). Although standard institutional and descriptive spider abbreviations are used whenever possible, they are defined here for the purposes of clarity: Institutional. AMNH (American Museum of Natural History), CAU (Auburn University, G. Mullen), CUC (Cornell University Collection, housed at AMNH), CDF (personal collection of D. Folkerts), JAB (personal collection of J. Beatty), ECU-AMNH (currently housed in the East Carolina University Spider collection, to be transferred to the AMNH), FSCA (Florida State Collection of Arthropods, G. Edwards), MCZ (Museum of Comparative Zoology, Harvard University, G. Giribet), USNM (United States National Museum, Smithsonian Institution, J. Coddington), VMNH (Virginia Museum of Natural History, R. Hoffman); Morphological. $\mathrm{Cl} / \mathrm{w}$ (carapace length/width), STR1/w (sternum length/width), LBl/w (labium length/ width), A/PER (anterior/posterior eye row), A/PME (anterior/posterior median eye), PL/ MS (posterior lateral/median spinnerets), TSp/ $\mathrm{r}$ and TSrd (male tibia I spines prolateral/ retrolateral and retrolateral distal), PTw/1 (male palpal tibia width/length), Bl (palpal bulb length), PT/TB3s (female patella/tibia III spines). Leg article measurements are in the following order: femur, patella, tibia, metatarsus, and tarsus. The variation sections document values measured from three individuals selected from multiple geographic locations to represent the extremes from all 


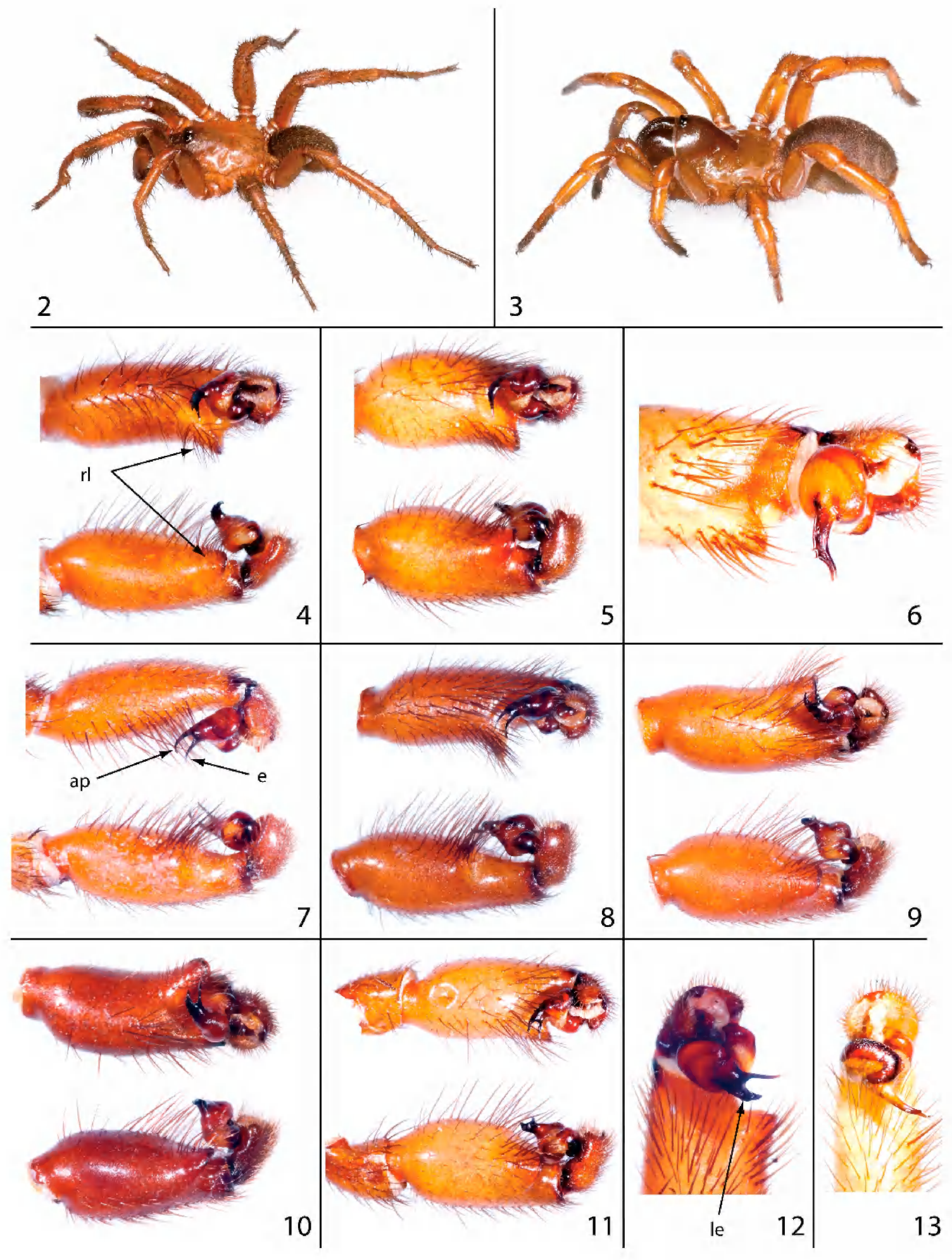


specimens examined (when sufficient material was available).

\section{SYSTEMATICS}

Family Cyrtaucheniidae Simon, 1892

Subfamily Euctenizinae Raven, 1985

Genus Myrmekiaphila Atkinson, 1886

Myrmekiaphila Atkinson, 1886: 131 (type species by monotypy Myrmekiaphila foliata Atkinson).

Myrmeciophila: Simon, 1891: 316 (invalid emendation).

Myrmekiophila: Petrunkevitch, 1929: 516 (invalid emendation).

Diagnosis: Males can be separated from those of the other euctenizine genera by the medially compressed and distally expanded metatarsus I (e,g,, fig, 14, arrow), All species other than $M$. minuta (fig. 13) have a retrolateral ledge on the male palpal tibia (e.g., fig. $4, \mathrm{rl}$ ) and a subdistally serrated palpal bulb and embolus (figs. 24-32). Females have distinctive spermathecae that are strongly sclerotized (at least basally) and bear cuticular outgrowths at their base (e.g., fig. 39) and can be distinguished from those of all other euctenizines by the presence of the following combination of characters: preening combs on metatarsi III and IV, a rastellum borne on a prominent mound with small rastellar spines, and carapace uniformly sclerotized. These spiders are known only from the southeastern United States.

DESCRIPTION: Small to moderately sized trapdoor spiders, carapace 2,80-8,00 long, 2.25-6.81 wide, glabrous or lightly hirsute, with fringe of stout black bristles ( $\hat{\delta}^{\prime} \mathrm{s}$ ) or lacking setae. Carapace coloration ranging from yellowish-red to dark reddish-brown (figs. 2, 3). Abdominal coloration uniform dark reddish-brown dorsally ( $d$ 's) or some species with wide dusky stripes on dorsum ( + 's, fig. 3), ventrum and spinnerets pale yellow. Carapace sclerotization uniform across its entire length. Foveal groove generally deep, procurved; straight to slightly recurved in some species. Eyes either not elevated or in some species elevated slightly on low mound. AME, PME subequal in diameter in majority of species; AER slightly procurved, PER slightly recurved. Pars cephalica moderately elevated (not steep).

Sternum, as in most euctenizines, wider posteriorly, tapering anteriorly with widest point at coxae III. Posterior pair of sternal sigilla usually much larger, in some species nearly contiguous. Palpal endites longer than wide, small patch of few (d's) or many ( $f$ 's) cuspules concentrated on proximal, inner margin. Labium wider than long or subquadrate, with few or no cuspules. Anterior margin of chelicerae with $7-12$ or more teeth, posterior margin with single row/patch of small denticles. Rastellum borne on distinct mound, armed with number of prominent spines, Anterior leg articles slender relative to posterior articles, lacking pseudosegmentation. Tarsi III, IV straight. Light to moderately dense scopulae on pedipalps ( ${ }^{\prime}$ 's) and legs I, II; light scopulae on tarsus of legs III, IV in some species. Tarsi I with single row of trichobothria, usually arranged in zigzag pattern, sometimes interspersed with setae. Preening combs at tarsus-metatarsus joint on legs III, IV ( $q$ 's), absent on leg III in some species.

Apical article of PLS digitiform, short; all three segments with fused spigots (see Bond and Opell, 2002: fig. 3H). Terminal segment with two or three enlarged spigots at tip. PMS single segment short, with spigots, terminus rounded.

Spermathecae relatively simple, comprising single pair of unbranched bulbs with moder-

Figs. 2-13. 2, 3. Live $M$. neilyoungi from Santa Rosa Co., Florida. 2. Male specimen. 3. Female specimen. 4-13. Color images of male pedipalps, ventral view (top of frame) and retrolateral view (bottom); 6, 12, 13 show ventral view only. 4. $M$. foliata, Knox Co. Tennessee ( $\mathrm{rl}=$ retrolateral ledge). 5. M. comstocki, Travis $\mathrm{Co}$, Texas. 6. $M$ coreyi, Thomas Co., Georgia. 7. M. fluviatilis, Marshall Co., Alabana (ap=accessory prong, e=embolus). 8. M. jenkinsi, Van Buren Co., Tennessee. 9. $M$. torreya, Liberty Co., Florida. 10. M. neilyoungi, Tuscaloosa Co., Alabama. 11. M. millerae, Grenada Co., Mississippi. 12. M. howelli, Newton Co., Mississippi (le=ledge), 13, M. minuta, Alachua Co,. Florida, 


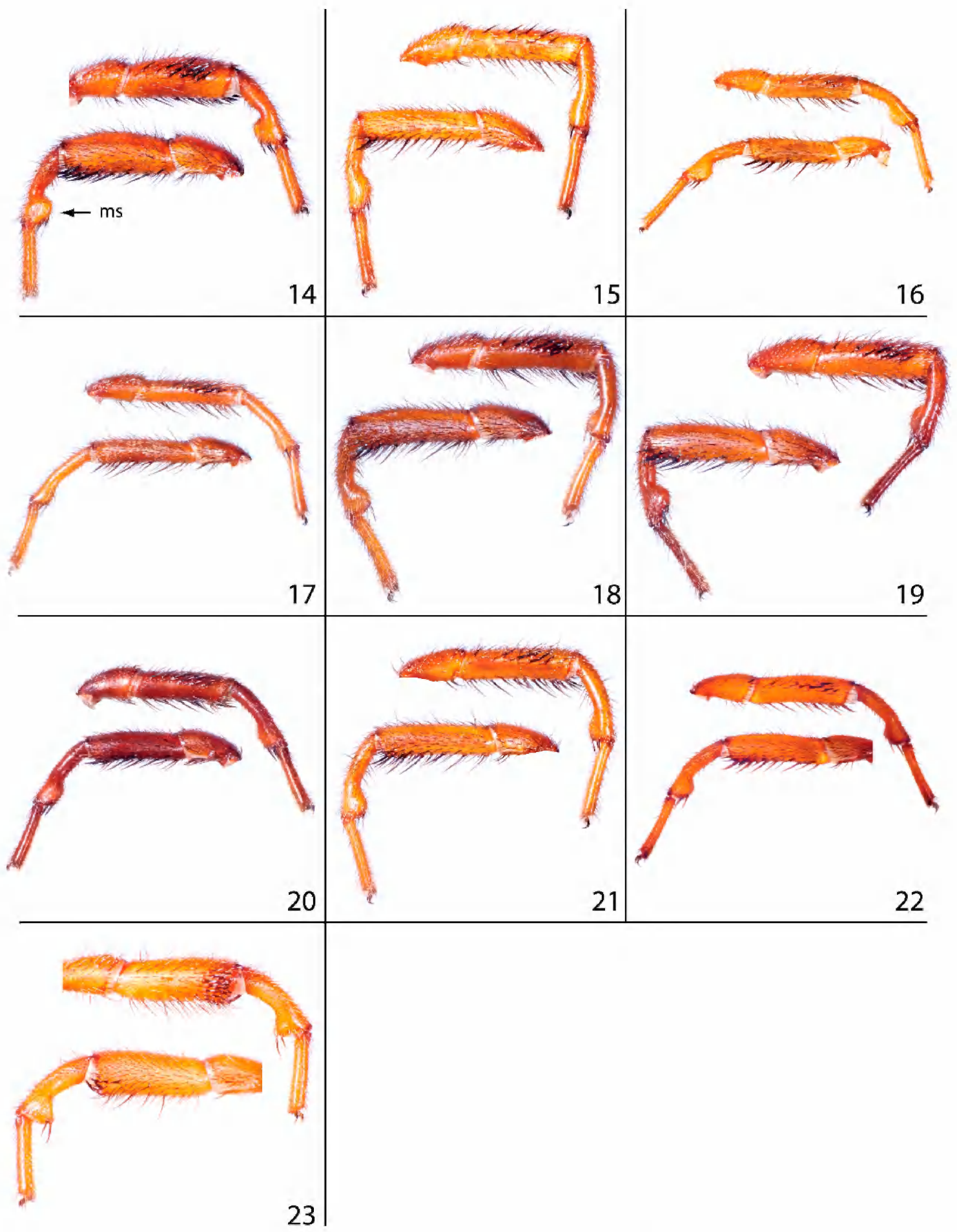

Figs. 14-23. Color images of male leg I mating claspers, prolateral (top of frame) and retrolateral views (bottom). 14. M. foliata, Knox Co., Tennessee (ms=metatarsal swelling). 15. M. comstocki, Travis Co., Texas. 16. M. coreyi, Thomas Co., Georgia. 17. M. fluviatilis, Marshall Co., Alabama. 18. M. jenkinsi, Van Buren Co., Tennessee. 19. M. torreya, Liberty Co., Florida. 20. M. neilyoungi, Tuscaloosa Co., Alabama. 21. M. millerae, Grenada Co., Mississippi. 22. M. howelli, Newton Co., Mississippi. 23. M. minuta, Alachua Co., Florida. 

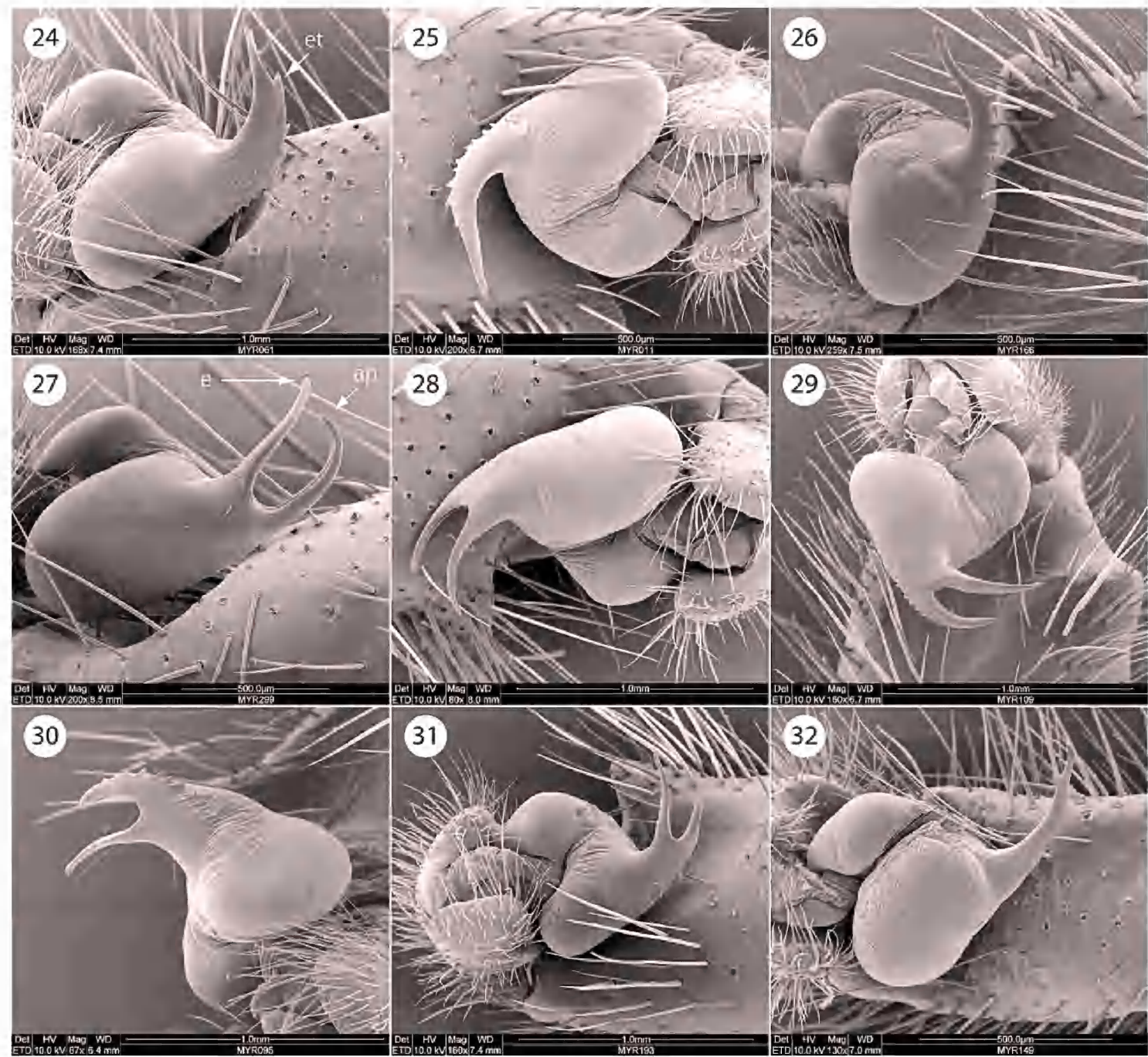

Figs. 24-32. Scanning electron micrographs of male palpal bulbs. 24. $M$. foliata, Knox Co,, Tennessee (et=embolus tooth). 25. $M$. comstocki, Travis Co., Texas. 26. $M$. coreyi, Jefferson Co., Florida. 27. $M$. fluviatilis, Marshall Co., Alabama ( $\mathrm{e}=$ embolus, ap=accessory prong). 28. M. jenkinsi, Van Buren Co., Tennessee. 29. M. torreya, Liberty Co., Florida. 30. M. neilyoungi, Jefferson Co., Alabama. 31. M. millerae, Grenada Co., Mississippi. 32. M. minuta, Alachua Co., Florida.

ately to heavily sclerotized base (e.g., figs. 39 , 47). Distinctive male mating clasper (modified leg I, e.g., fig. 14); metatarsus medially compressed, expanded at metatarsus-tarsus junction. Cymbium of male pedipalp lacks spines, embolus with serrations or single tooth, some species with secondary prong (figs. 24-32).

Distribution: Southeastern United States, extending westward into the Midwest and eastern Texas.
Species Groups: We recognize three species groups for the purposes of facilitating identification. Because a phylogeny of the genus is not available at present, these informal groups are not intended to represent monophyletic taxa. The foliata group contains three species in which the male palp bears no second prong, having at most a single enlarged tooth or serration. The fluviatilis group contains six species in which the male palp bears 

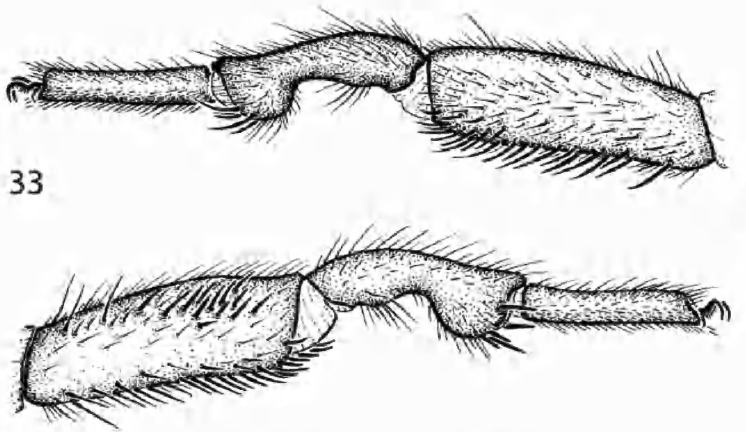

34

35
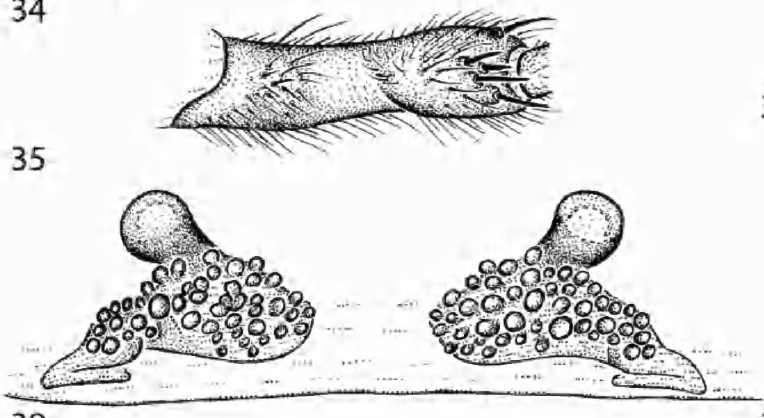

39

\section{8}
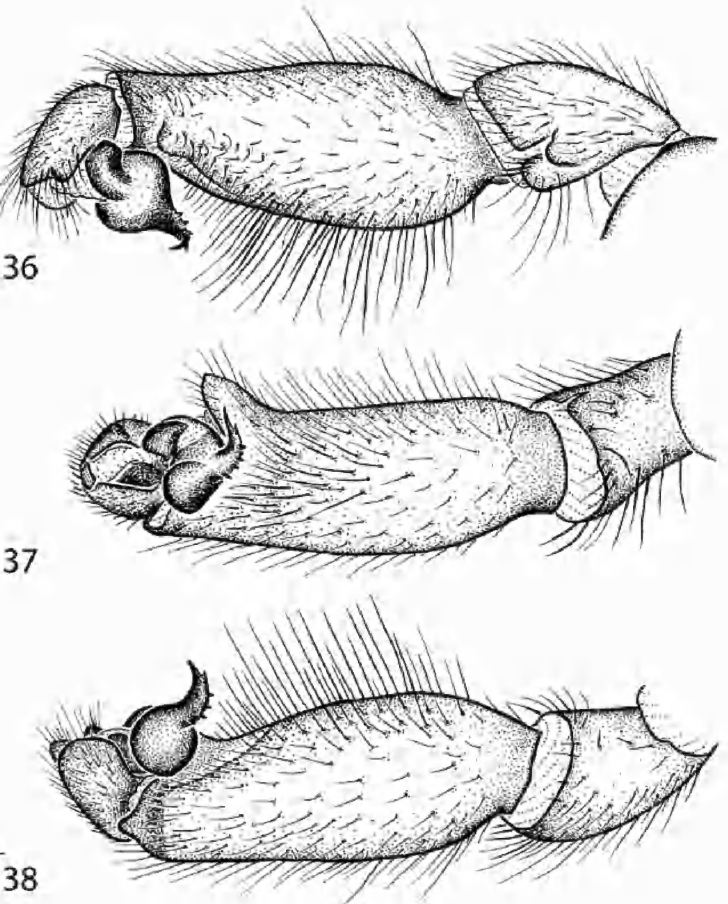

Figs. 33-39. Myrmekiaphila foliata (Hentz) from Mercer Co., West Virginia. 33-35. Male left leg I: retrolateral, prolateral, and ventral (metarsus) views. 36-38. Male left pedipalp: retrolateral, ventral, and prolateral views. 39. Spermathecae.

a second prong. The minuta group comprises one species in which the male palp bears no second prong, having a vestige of a single embolar tooth situated distally rather than proximally, and lacking a retrolateral ledge on the palpal tibia. One species known only from females remains unplaced.

\section{Key to Males}

1. Palpal tibia with retrolateral ledge (rl, fig. 4). 2 Palpal tibia without retrolateral ledge (fig. 102) . . . . . . . . . . . M. minuta

2. Palpal bulb with embolus only, lacking accessory prong (figs. 4-6) . . . . . . . . 3

Palpal bulb with embolus and accessory prong (ap, figs. 7-12) . . . . . . . . . 5

3. Distal dilation of metatarsus I small (fig. 15), palpal tibia usually short and robust, embolus lacking a prominent ledge (figs. 5,25 ) . $\ldots \ldots \ldots \ldots \ldots . \ldots$. comstocki

Distal dilation of metatarsus I large (fig. 14) . . 4

4. Embolus thick, with enlarged, retrolaterally directed subdistal tooth or ledge (figs. 4, 24), metatarsal dilation typically with more than two ventral spines (fig. 35); palpal tibia generally slender (figs. 4,36 ) .... M. foliata

Embolus very thin, with or without enlarged subdistal tooth (if enlarged, tooth directed somewhat proximally, with wide angle between tooth and embolus); palpal tibia short (figs. $6,26,52) \ldots \ldots \ldots \ldots$. . corey $i$

5. Accessory prong much shorter than embolus (figs. 9, 29) . . . . . . . . . . 6

Prong subequal in length to embolus (figs. 7 , 27)...................... . . 9

6. Prong with accessory ledge below, appearing doubled distally (fig. 12, le) .... M. howelli

Prong without such a ledge (fig. 7). . . . . 7

7. Prong typically ending in sharp point, tip pointing straight out or back up to embolus (fig. 9) . . . . . . . . . M. torreya

Prong with thick, curved tip, with or without tooth before curve (figs, 10,11 ) . . . . 8

8. Palpal tibia with retrolateral excavation, retrolateral ledge elongated and slender (fig. 10) ............... neilyoungi

Palpal tibia without retrolateral excavation, retrolateral ledge short and stubby (fig. 11) ............... millerae

9. Prong and embolus subequal, prong weakly curved; palpal tibia usually slender, with 


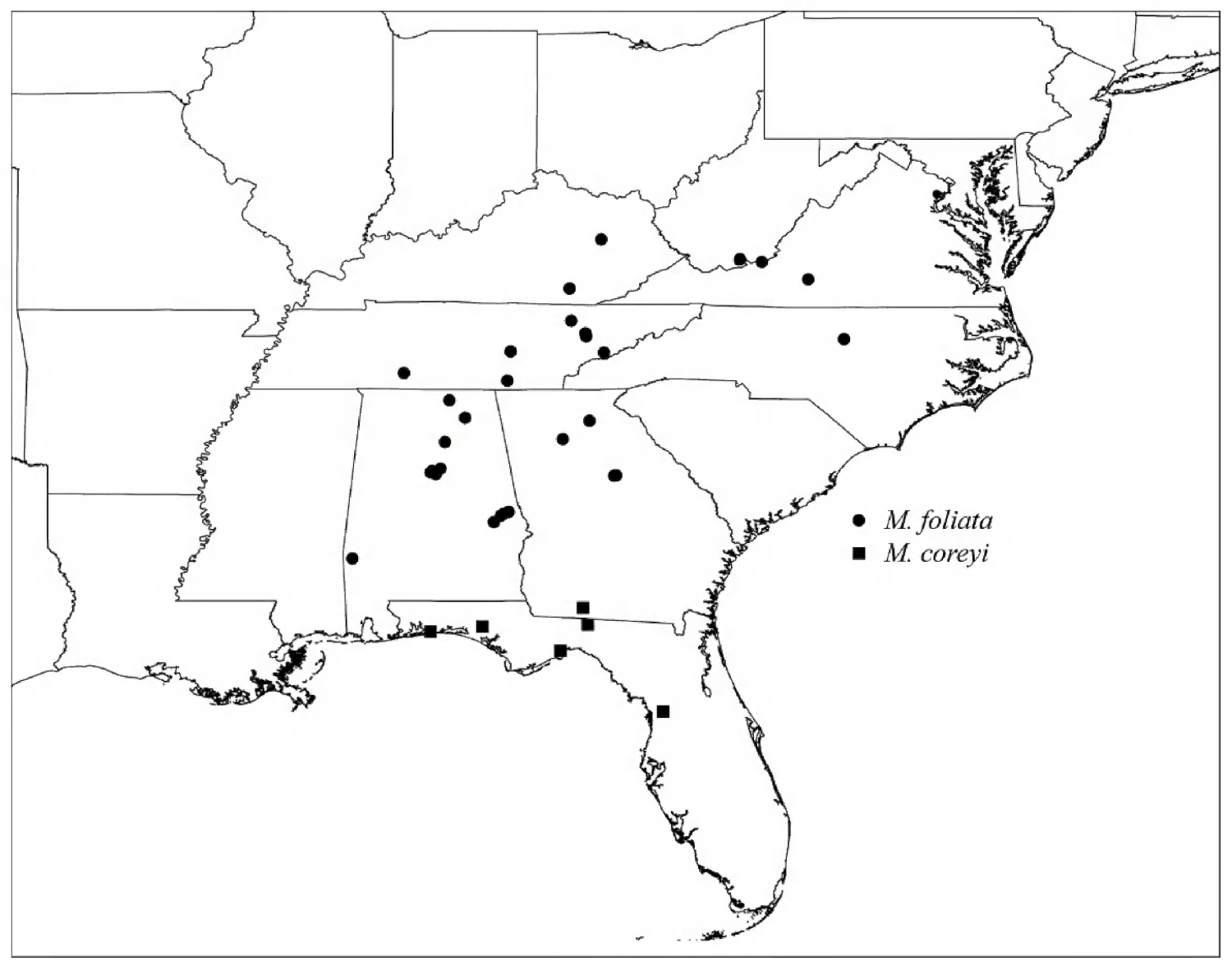

Fig. 40. Records of M. foliata (Hentz) and M. coreyi n. sp.

retrolateral ledge situated more proximally (fig. 8) ................. jenkinsi Prong slightly shorter than embolus, prong strongly curved; palpal tibia usually thicker, with retrolateral ledge situated distally (fig. 7) .............. fluviatilis

\section{The FOLIATA Group}

\section{Myrmekiaphila foliata Atkinson}

Figures 4, 14, 24, 33-40

Myrmekiaphila foliata Atkinson, 1886: 132 (three female syntypes from Chapel Hill, Orange Co., North Carolina, in CUC and USNM, examined). Myrmeciophila atkinsoni Simon, 1891: 316 (female holotype from Occoquan Falls, Prince William Co., Virginia, in USNM, examined). NEW SYNONYMY.

Myrmekiaphila fluviatilis (misidentification): Bishop and Crosby, 1926: 166, figs. 1-6.
DiAgnosis: Males can be recognized by the enlarged, retrolaterally directed, subdistal tooth on the embolus (fig. 24) and the relatively large distal dilation on metatarsus I, usually bearing more than two ventral spines (figs. 33-35), females by the triangular spermathecal bases and by the spermathecal bulbs originating from the median side of the spermathecal bases (fig. 39).

DESCRIPTIONS: Male described (as $M$. $f l u$ viatilis) by Bishop and Crosby (1926); female described by Atkinson (1886) and (as $M$. atkinsoni) by Simon (1891).

MATERIAL EXAMINED: UNITED STATES: ALABAMA: no locality data [MYR084], (R. Jenkins, W. Howell, AMNH), 1 है. Blount Co.: no specific locality [33.9668 -86.5833 , MYR301], Mar. 14, 1998 (AMNH), 1 ㅇ. Choctaw Co.: Silas [31.7654 -88.329, MYR013], 
Feb. 19, 1912 (H. Smith, CUC), 12. Jefferson Co.: Linda Drive, Bluff Park [33.3935 -86.85306, MYR004], Dec. 25, 1996 (W. Howell, AMNH), 28; Tyler Road [33,4152 -86,8261, MYR225], Nov, 26, 1996 (R. Jenkins, W. Howell, AMNH), I 3 . Lee Co: Opelika [32.6454-85.3783, MYR133], Jan. 10, 1985 (D. Folkerts, CDF), 1\&; 2 mi S Auburn along Wire Road [32.5776 -85.5246, MYR135], Feb. 24, 1974 (R. Skinner, CAU), 18; Auburn [32.6099 -85.4808, MYR 124], (AMNH), 2\%, 1 juv.. [MYR 127], (N. Banks, C. Baker, MCZ), 1年, [MYR [32], Apr. 10, 1941 (AMNH), 39, [MYR 137], Mar. 3, 1968 (W. Ivey, CAU), 18, [MYRI39], May 10, 1975 (B. Muse, CAU), 1ㅇ․ [MYR288], June 1, 1986 (G. Mullen, CAU), 1․ Macon Co.: Tuskegee National Forest, Wire Road, S Interstate 85 [32.4577 -85.6576, MYR 138], Dec. 12, 1975 (Weatherley, Brooks, CAU), 1.. Madison Co.: Monte Sano State Park, 34.74599-86.50653 [MY2025], June 11, 2003 (B. Hendrixson, P. Marek, ECU-AMNH), 1.. Montgomery Co.: McGus Station [MYR140], Oct. 24, 1915 (H. Smith, CUC), 18. Shelby Co.: Lake Purdy Dam [33.4598 -86.6694, MYR088], Feb. 3, 1997 (R. Jenkins, W. Howell, AMNH), 1\%; Indian Springs [33.359-86.7566, MYR024], Nov. 14, 1997 (T. Honea, AMNH), Tuscaloosa Co: University [MYR086], Nov. 29, 1910 (H. Smith, AMNH), 18 . GEORGIA: Hall Co: Thompson Mill [34.3665 -83,8588, MYR 141], (M. Allen, MCZ), 19. Putnam Co.: no specific locality [33.3335 -83.3499, MYR040], Apr. 23, 1974 (W. Merrill, FSCA) 18; Eatonton [33.3268 -83.3885, MYR 131], Apr. 18, 1974 (W. Merrill, FSCA), 18. KENTUCKY: Wolfe Co: Koomer Ridge [37.7904-83.633, MYR129], Apr. 28, 1968 (J. Tripp, F. Moore, FSCA), 1․ Whitley Co.: $0.1 \mathrm{mi}$ from junction of US-25W on KY -90 , in very disturbed ravine, 36.85428 84.23483, elev, $385 \mathrm{~m}$ [MY2182], Aug, 10, 2003 (B. Hendrixson, ECU-AMNH), I juv. NORTH CAROLINA: Orange Co.: Chapel Hill [35.9071 -79.0483, MYR 119], Mar. 13, 1886 (G. Atkinson, CUC), 17, [MYR 120]. Apr. 1, 1886 (G. Atkinson, USNM), 1t, [MYRI21], Dec. 31, 1929 (J. Beakley, AMNH), 18, [MYR 122], (G. Atkinson, AMNH), 1 \% [MYR126], Nov. 1. 1983 (AMNH), $3 \approx$.
TENNESSE: Campbell Co: $2.6 \mathrm{mi}$ NW Route 116 on Beech Grove Road, 36.23878 -84.19148, elev. $300 \mathrm{~m}$ [MY2180], Aug. 10, 2003 (B. Hendrixson, ECU-AMNH), 1 ?. Knox Co.: Knoxville $\left[\begin{array}{ll}35.9606 & -83.9207 .\end{array}\right.$ MYR038], Nov. 4, 1985 (T. Stevens, AMNH), 1है, [MYR 118], Mar. 4, 1920 (W. Cartwright, CUC), 1; Powell, Riechert Property, ca $0.11 \mathrm{~km}$ NNW intersection of Cruze Drive and Bell Campground Road [36.03098 -84.06139, MYR019, 021, 026, 028-030, 036, 059-075, 125], elev, $348 \mathrm{~m}$, Apr.-Nov 1985 (S. Riechert, AMNH), $44 \star$. Lawrence Co.: David Crockett State Park, 35.26252 -87.36217, elev. 278 m [MY2801], May 2, 2004 (B. Hendrixson, ECU-AMNH), I . Marion Co.: Stanley [35.1226 -85.4039 , MYR087], (AMNH), 18 . Sevier Co.: Elkmont [35.6537 -83.5805, MYR130], May 14, 1967 (F. Moore, FSCA), 29. Van Buren Co.: Fall Creek Falls State Park, along Cane Creek Cascade Trail, 35.66182 -85.34962, elev. $500 \mathrm{~m}$ [MY2175], Aug, 9, 2003 (B. Hendrixson, ECU-AMNH), 1 juv. VIRGINIA: Franklin Co:: Smith Mountain Lake [37.0371 -79.7238, MYR134], Oct. 7. 1994 (G. Atkinson, AMNH), 13 \%. Giles Co.: Cascades Recreation Area, small creek near main trailhead, $37.35383-80.59988$, elev. $670 \mathrm{~m}$ [MY2235], Aug. 15, 2004 (B, Hendrixson, ECU-AMNH), 1 juv. Prince William Co.: Occoquan Falls [38.68363 -77.26056, MYR117], (G. MarX, USNM), 1․ WEST VIRGINIA: Mercer Co.: Athens, Old Pumphouse [37.4223 -81.01638, MYR128], May 17, 1966 (O. Pendleton, B. Armes, AMNH), 19;2 mi S Athens [37.3931 -81.016185, MYR 194], Sept. 18, 1965 (W. Shear, AMNH), 18.

Distribution: Southeastern United States, from northern Virginia south to central Alabama and northern Georgia (fig. 40).

SYNonymy: Banks $(1900 ; 530)$ placed $M$. foliata as a junior synonym of $M$. fluviatilis (Hentz); based on the material examined by us, that synonymy appears to be crroncous. Simon (1891) did not examine the genitalic characters of $M$. foliata or $M$. atkinsoni, separating the taxa only by labial cuspule, leg spination, and tarsal claw dentition; these are differences that can vary between the left and right sides of a single specimen. 

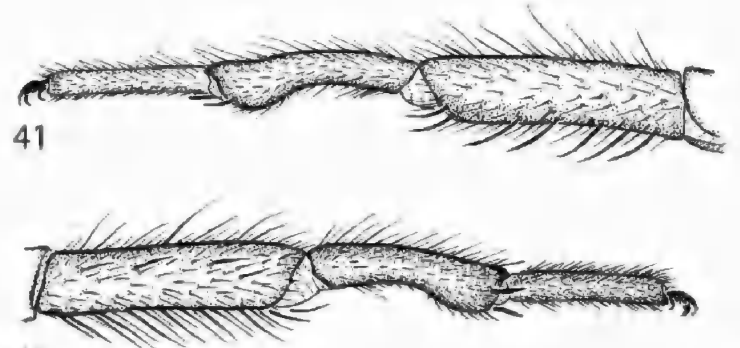

42
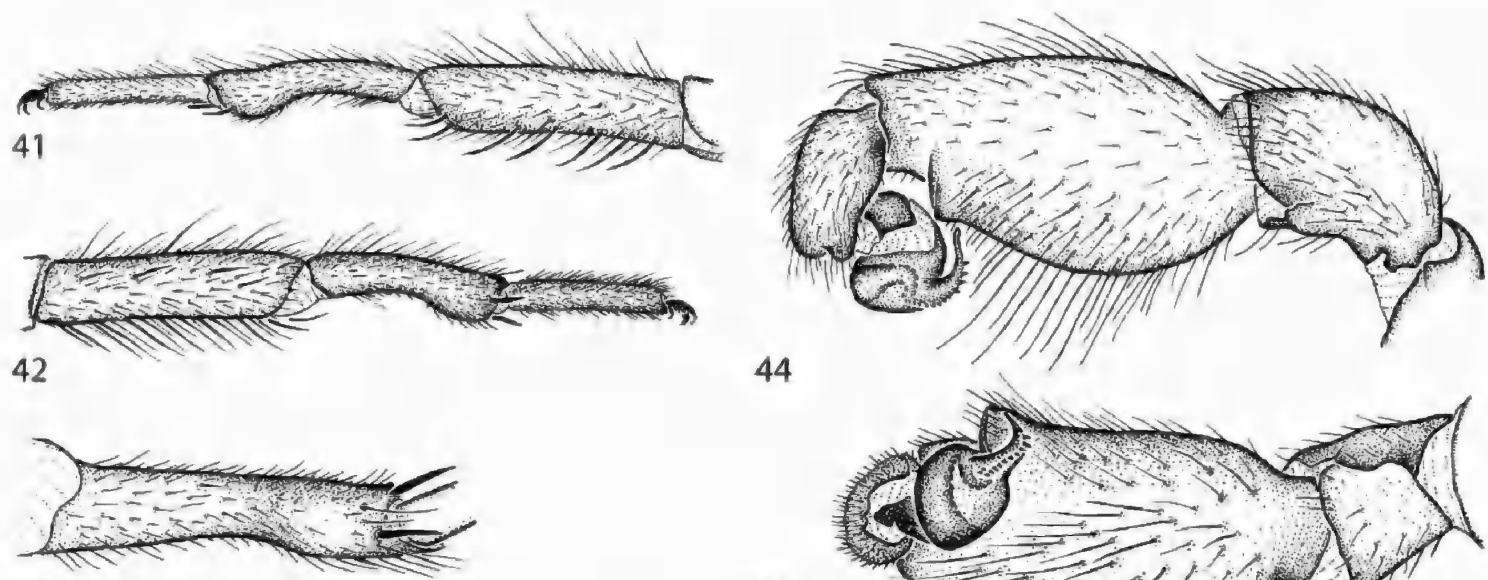

43

45
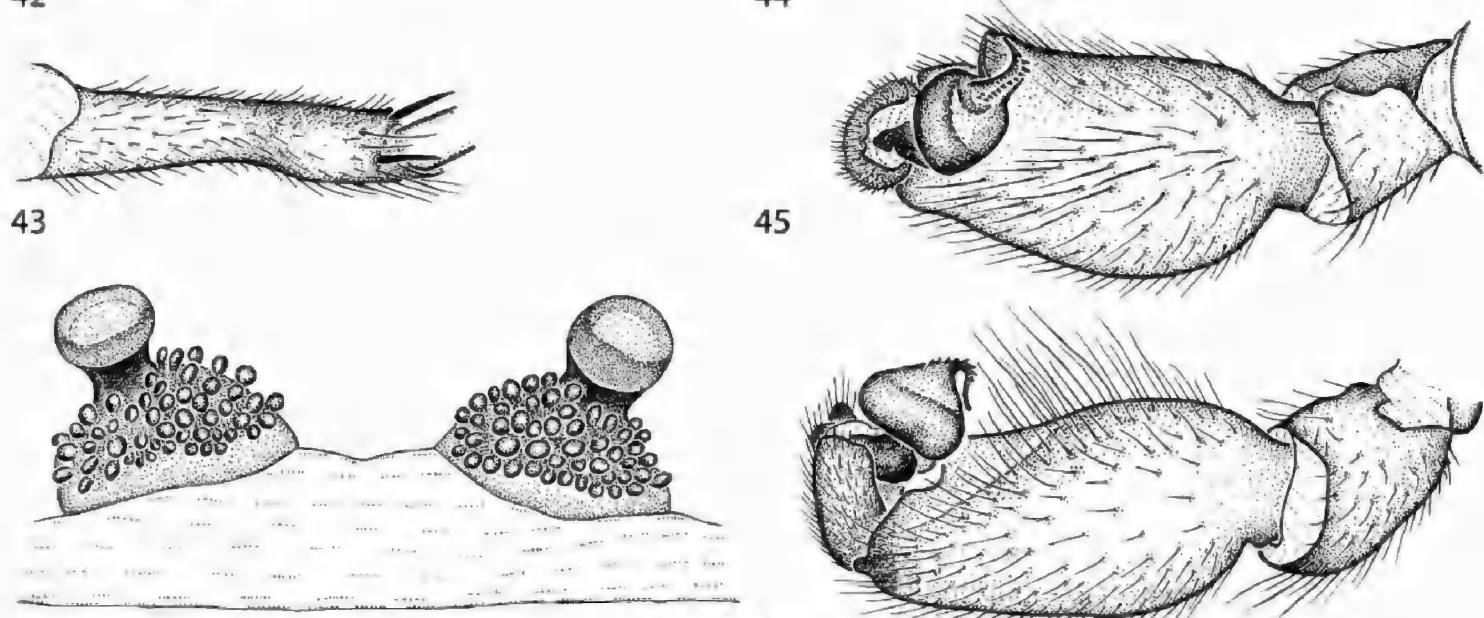

47

46

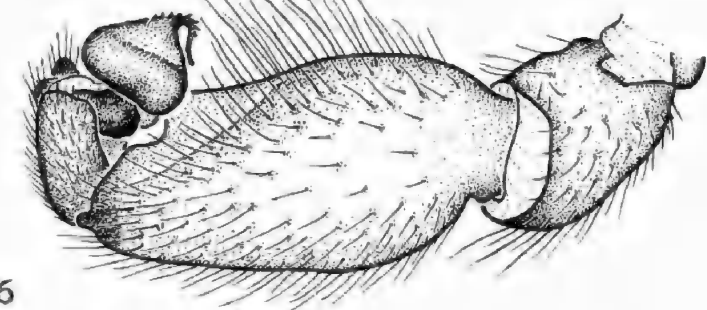

Figs. 41-47. Myrmekiaphila comstocki Bishop and Crosby from Travis Co., Texas. 41-43. Male left leg I: retrolateral, prolateral, and ventral (metarsus) views. 44-46. Male left pedipalp: retrolateral, ventral, and prolateral views. 47. Spermathecae.

Myrmekiaphila comstocki Bishop and Crosby

Figures 5, 15, 25, $41-48$

Myrmekiaphila comstocki Bishop and Crosby, 1926: 168.169 (male holotype from Austin, Travis Co., Texas, in AMNH, examined).

Myrmekiophila fluviatilis (misidentification): Petrunkevitch, 1929: 516.

Diagnosis: Males can be distinguished by the unbranched palpal bulb with the embolus lacking a prominent distal tooth (fig. 25), the robust palpal tibia (figs. $5,44-46$, not as slender as that of $M$. foliata), and by having metatarsal dilation of leg I less prominent than in other species (figs. 15, 41-43). Female $M$. comstocki and $M$. foliata specimens are difficult to distinguish on the basis of subtle spermathecal differences (fig. 47); however, the distributions of these two species do not overlap (fig. 1).
Descriptions: Male described by Bishop and Crosby (1926); female described by Gertsch (1935).

Material Examined: UNITED STATES: ARKANSAS: Bradley Co.: no specific locality [33.47 -92.16, MYR076], Nov. 9, 1963 (AMNH), 1 $\hat{\text { A }}$. Dallas Co.: $4 \mathrm{mi} \mathrm{S}$ Dalark [34.0680 -93.06417, MYR077, 078], June 10, 1973 (J. Rowland, AMNH), 2 \% . Montgomery Co.: 1 mi S State Road 8 on FS road [34.4502 -93.8887, MYR289], June 28, 1972 (F. Coyle, AMNH), 1 \% . Pike Co.: Glenwood [34.3268 -93.5507, MYR018], Jan. 8, 1954 (N. Causey, MCZ), 1 f . Polk Co.: Sugar Creek, FS Road 38 , ca $2.5 \mathrm{mi} \mathrm{S}$ intersection with FS Road 25 [34.4322 -94.107, MYR041-46], June 18, 1972 (F. Coyle, AMNH), 4q, 1 eggsac; Ouachita National Forest, Caney Creek WMA on CR 81 near Sugar Creek, 34.42985 -94.13922, elev. 313 m [MY3387-3389], July 9, 2005 (B. Hendrixson, ECU-AMNH), 3 ㅇ. Washington 


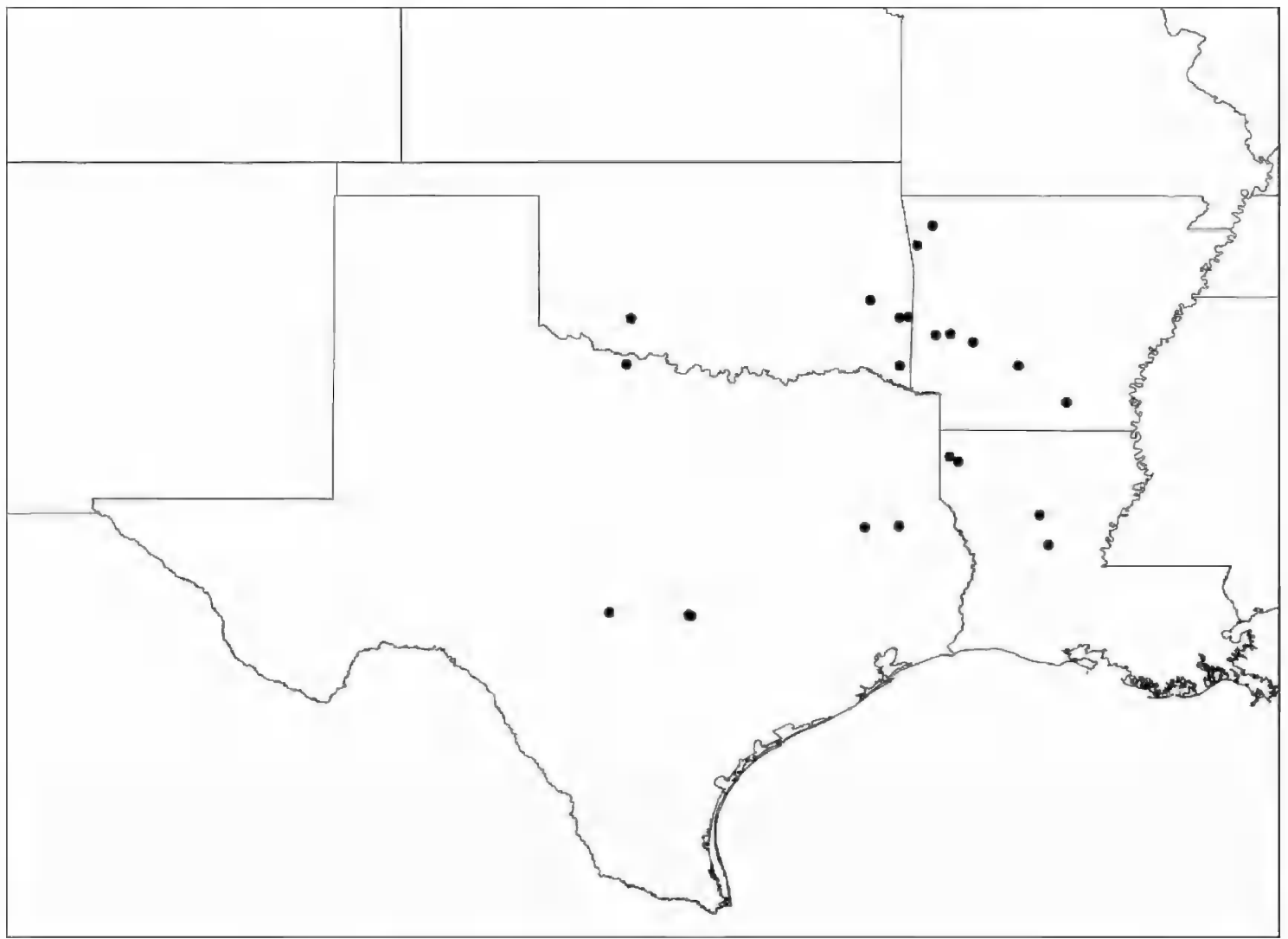

Fig. 48. Records of M. comstocki Bishop and Crosby.

Co.: Fayetteville [36.0626-94.1574, MYR016], Nov. 1, 1962 (W. Whitcomb, AMNH), 1 है; Cove Creek [35.7655 -94.3829, MYR037], Apr. 27, 1962 (M. Hite, AMNH), 1 \%, [MYR023], Oct. 20, 1962 (O., M. Hite, AMNH), 20ं; Cove Creek Valley, $15 \mathrm{mi} \mathrm{S}$ Prairie Grove, Boston Mountains [MYR039], Sept.-Oct. 1955 (M. Hite, AMNH), 1 ô, 1 juv., [MYR027], Mar. 1, 1957 (M Hite, MCZ), 1 \&ै. LOUISIANA: Caddo Par.: no specific locality [32.6335 -93.9002, MYR057], Nov. 21, 1936 (R. Bellamy, AMNH), $1 \%$; Shreveport [32.55813 -93.7756, MYR056], Oct. 13, 1936 (R. Bellamy. AMNH), 1 ㅇ. Grant Par.: Kisatchie National Forest [31.7668 -92.5668, MYR012], June 1941 (Jones, A. Archer, AMNH), $1 \tilde{d}$. Rapides Par.: Pineville [31.3224 -92.4343, MYR055], Dec. 31, 1976 (J. Weaver, AMNH), 1 . OKLAHOMA: Comanche Co.: Wichita Mountains [34.6829 -98.6331, MYR292], Mar. 25, 1978 (F. Bryce, AMNH), $10{ }^{\AA}$. La Flore Co.: on Route $2705.5 \mathrm{mi} \mathrm{E}$ intersection with Route 259 [34.7048 -94.5143, MYR048], June 27, 1972 (F. Coyle, AMNH), 1; Pipe Spring on Route 259, $1.5 \mathrm{mi} \mathrm{S}$ intersection with Route 1 [34.691 -94.6453, MYR047], June 27, 1972 (F. Coyle, AMNH), 19. Latimer Co.: Red Oak [34.9509 -95.0802, MYR031], Oct. 1, 1975 (K. Stephan, AMNH), 6̊; Red Oak [34.7048 -94.5143, MYR049], Oct. 8, 1976 (V. Roth, AMNH), 1 \% . McCurtain Co.: 5 mi SW Eagletown [33.9771 -94.639, MYR050], Aug. 7, 1946 (B. Branson, AMNH), 1 ㅇ. TEXAS: Cherokee Co.: Alto [31.5782 -95.16583, MYR005], May 15, 1979 , (F. Moody, AMNH), 1 ㅇ․ Kimble Co.: (mislabeled as Gillespie Co.) Junction [30.4886 -98.9503, MYR035], Apr. 14, 1979.(T. Hall, AMNH), 1 \&. Nacogdoches Co.: Nacogdoches [31.6035-94.6555, MYR010], Oct. 14, 1968 (H. McDonald, AMNH), $1 \delta$. Travis Co.: Austin [30.2669-97.7429, MYR009], May 21, 1913 (C. Hartman, AMNH), 1 \%, 1 juv.; Brackenridge Field Laboratory [30.2833 -97.7787, MYR011, 

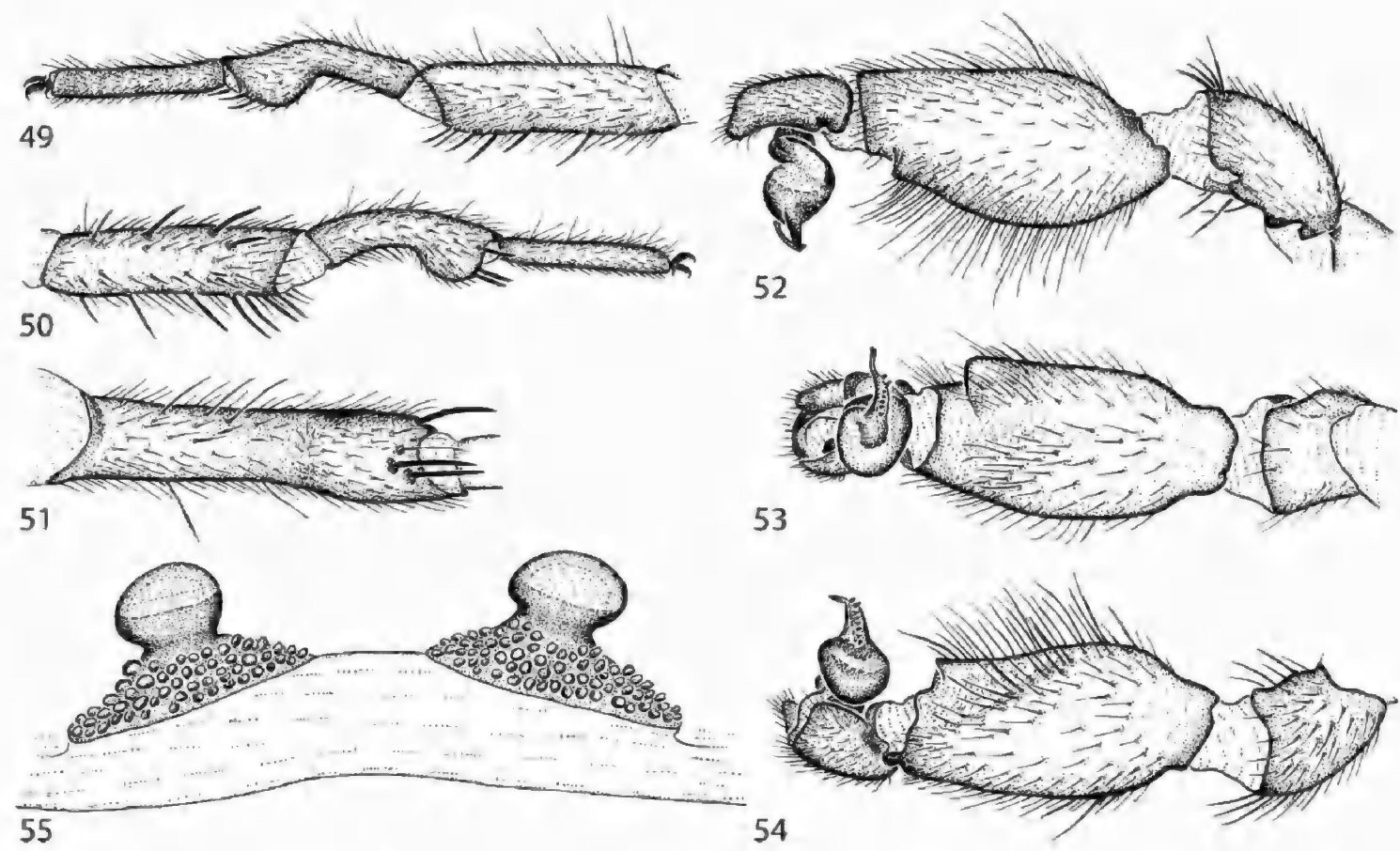

Figs. 49-55. Myrmekiaphila coreyi, new species, from Thomas Co., Georgia (male) and Citrus Co.. Florida (female). 49-51. Male left leg I: retrolateral, prolateral, and ventral (metarsus) views. 52-54. Male left pedipalp: retrolateral, ventral, and prolateral views. 55. Spermathecae.

014, 020, 025, 032-034, 290, 291], Jan.-Apr 1979 (B. Vogel, AMNH), 88 fै, 2 ㅇ, 1 juv. Wichita Co.: no specific locality $[34.0-98.7$, MYR006], Mar. 1, 1973 (Hicks, AMNH), $1 \delta$, [34.0 -98.7, MYR017], Mar. 1, 1973 (T. Salmon, AMNH), $1 \hat{\text { ô. }}$.

Distribution: Widely distributed throughout Arkansas, Louisiana, Okalahoma, and eastern/central Texas (fig. 48).

\section{Myrmekiaphila coreyi, new species}

Figures 6, 16, 26, 40, 49-55

TyPes: Male holotype and female paratype from St. Mark's National Wildlife Refuge - Panacea Unit, $1 \mathrm{mi}$ SW Panacea [30.0241 -84.404], Wakulla Co., Florida (FebMar. 1978), deposited in AMNH.

Etrmology: The specific name is a patronym in honor of Dr. David Corey.

Diagnosis: Males can be distinguished by the presence of a very slender unbranched embolus with prominent serrations (figs, 6,
26) and a retrolateral ledge on the palpal tibia (figs. 52-54). Females have a subtriangular spermathecal base with a spermathecal bulb originating from the center of the base, constricted medially, and lacking a distinct stalk (fig. 55).

MALE (hOLOTYPE): Specimen preparation and condition. Specimen collected in live pitfall trap, preserved in ca. 70\% ethanol. Coloration faded. Pedipalp, leg I left side removed and stored in vial with specimen. General coloration. Carapace, chelicerae, and legs yellowish red (5YR 4/6). Abdomen uniform dark reddish brown (5YR 3/4) dorsally, ventrum and spinnerets pale yellow. Cephalothorax. Carapace 4.56 long, 3.84 wide, glabrous, stout black bristles along fringe; surface smooth, pars cephalica elevated. Fringe, posterior margin with black bristles. Foveal groove deep, moderately procurved. Eyes on low mound. AER slightly procurved, PER slightly recurved, PME, AME subequal in diameter. Sternum moderately setose, STR1 2.32, STRw 2.20. Posterior sternal sigilla large, positioned centrally, not contiguous, anterior sigilla pairs small, oval, marginal. 
Chelicerae with distinct anterior tooth row (11 teeth), posterior margin with single row of small denticles. Palpal endites with patch of small cuspules on proximal, inner margin, labium lacks cuspules, LBw 0.75, LBl 0.47. Rastellum consists of four or five very stout spines on prominent mound. Abdomen. Setose, heavy black setae intermingled with fine black setae. Leg I: 4.24, 2.04, 3.24, 2.40, 2.00; leg IV: 4.40 , $1.80,3.80,3.76,2.40$. Light tarsal scopulae on legs I, II. Tarsus I with single, slightly staggered row of 7 trichobothria. Leg I spination pattern illustrated in Figures 16, 49-51; TSp 6. TSr 5, TSrd 1. Pedipalp. Articles stout, lacking distinct spines (figs. 52-54). PTw 0.92, PTl 1 .87, Bl 0.80. Ledge on distal-retrolateral surface of palpal tibia (figs. 52, 53). Embolus slender, tapering sharply toward tip, with serrations (figs. 6, 26).

Variation (3). Cl 4.00-4.56, Cw 3.40-3.84; STRl 2.32-2.36, STRW 2.20-2.36; LBw 0.75. LBI $0.375-0.47$; leg I: $3.604 .24,1.56-2.04$, $2.68-3.24,2.08-2.40,1.96-2.00$; leg IV: 3.60 $4.40,1.60-1.80,3.20-3.80,3.28-3.76,2.20$ 2.40; PTw 0.85-1.00, PTl 1.62-1.87, Bl 0.75 0.80: TSp 4 6, TSr 5-6.

FEMALE (PARATYPE): Specimen preparation and condition. Female collected from live pitfall trap, prepared in same manner as male holotype. Genital plate removed and stored in microvial with specimen. Color. Carapace, legs, chelicerae, dark reddish brown (2.5YR $2.5 / 4$ ). Abdomen uniform dark reddish brown dorsally (5YR 2.5/2), ventrum and spinnerets pale yellow: recently collected specimens have wide dusky stripes on dorsum of abdomen. Cephalothorax. Carapace 4.80 long, 3.68 wide, glabrous; generally smooth surface, pars cephalica moderately elevated. Fringe lacks setae. Foveal groove deep, slightly procurved. Eye group slightly elevated on low mound. AER slightly procurved, PER slightly recurved, PME, AME subequal in diameter. Sternum moderately setose, STRI 2.60, STRw 2.52. Three pairs of sternal sigilla, anterior pairs small, oval, marginal, posterior pair much larger, oval, mesially positioned. Chelicerae anterior tooth row armed with nine tecth, posterior margin with denticle patch. Palpal endites with cuspules [ca. 30] concentrated at inner (promargin) posterior heel; labium with four cuspules, LBw $0.87, \mathrm{LB}$ 0,50 . Rastellum consisting of two very stout spines positioned on mound; fringe of short spines along distal promargin extending upward from rastellum. Abdomen. Moderately setose. Legs. Leg I: 3.16, I.72, 2.00, 1.44, 1.00; leg IV: $3.16,1.64,2.40,2.20,1.32$. Tarsus I with single staggered row of seven trichobothria. Legs I III with moderately light scopulae on tarsi only. PT3s 15, TB3s 0. Distinct preening comb on retrolateral distal surface (at tarsus-metatarsus joint) of metatarsi III. IV. Spermathecae. Two simple spermathecal bulbs, lacking elongate neck, arranged on subtriangular spermathecal base (fig. 55).

Variation (3). Cl 4.56-6.00, CW 3.68-4.40; STRI 2.56-3.40, STRw 2.40-3.08: LBw 0.850.97 , LBI $0.50-0.57$; leg I: $3.16-3.72,1.72-3.00$, $2.00-2.56,1.44-2.56,1.00-1.40$; leg IV: 3.16 $3.40,1.64-2.20,2.40-3.00,2.20-2.40,1.32-1.40$.

MATERIAL, EXAMINED: UNITED STATES: FLORIDA: Citrus Co.: no specific locality [28.8747 -82.4581, MYR201], Feb. 26, 1937 (H. Wallace, AMNH), 37. I juv. Jefferson Co: no specific locality $\left[\begin{array}{lll}30.516 & -83.888 \text {, }\end{array}\right.$ MYR007, 008, 015, 165-168], Jan.-Mar 1969 (AMNH), 98; Big Bend Horticultural Lab [MYR305]. Mar, 10, 1969 (AMNH), 1 है. Santa Rosa Co.: Santa Rosa Island [30.3824 -86.8564, MYR 170], Apr. 5, 1934 (H. Wallace, AMNH), I juv. Wakulla Co.: St. Mark's National Wildlife Refuge-Panacea Unit, 1 mile SW Panacea $[30.0241-84.404$, MYR022, $156-$ 162, 228, 293]. Feb.-Mar. 1978 (AMNH), 58 \& . 27. Washington Co.: 1-2 mi N FL-20 on FL79. near cemetery, $30.46376 \quad 85,85,86335$ [MY2557-2562], Feb. 27, 2004 (B. Hendrixson, D. Beamer, E(U-AMNH), 6 ? . GEORGIA: Thomas Co.: Boston, Bar M Ranch [30.7919 -83.7900, MYRI69]. Apr. 1969 (W. Sedgwick, MCZ), 18, juv. Thomas Co.: near Thomasville $[30.8366-83.9788$, MYR202], 1968 (AMNH), 1 \& .

Distribution: Known only from Florida and far southern Georgia (fig. 40).

\section{The flemiatilis Group}

Myrmekiaphila fluviatis (Hentz)

Figures $7.17 .27,56-62$

Mygale fluviarilis Hentz. 1850: 286 (female holotype from Tennessee River, Alabama, destroyed).

Bolostromus fluriatilis: Banks, 1892: 147.

Myrneciophila fluviatilis: Banks, 1900; 530. 

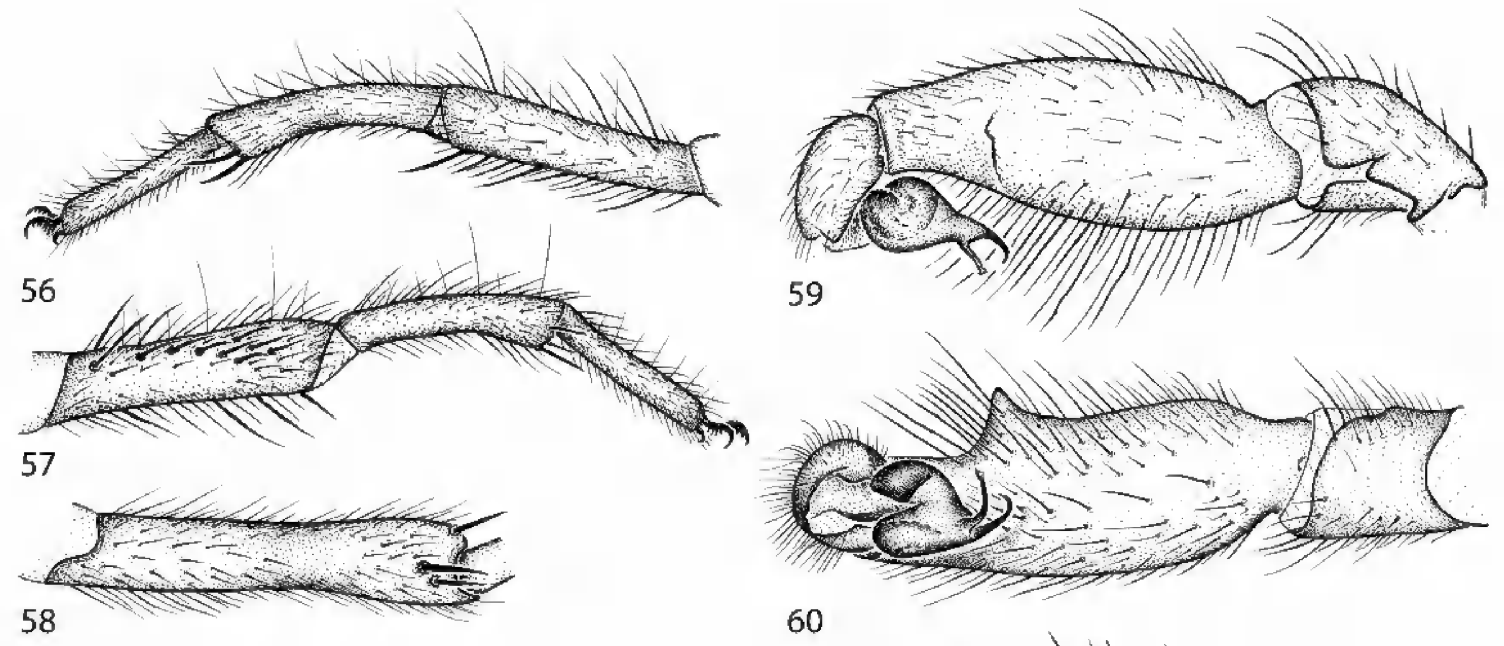

60

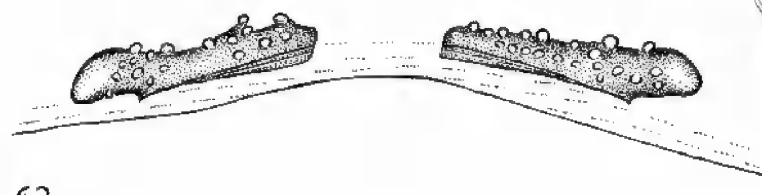

62

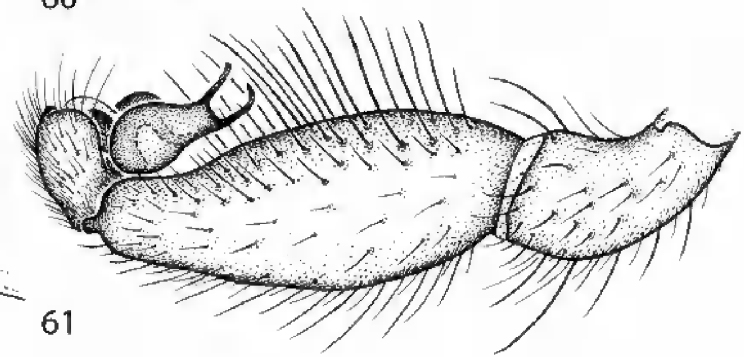

Figs. 56-62. Myrmekiaphila fluviatilis (Hentz) from Marshal Co. (male) and DeKalb Co. (female), Alabama. 56-58. Male left leg I: retrolateral, prolateral, and ventral (metarsus) views. 59-61. Male left pedipalp: retrolateral, ventral, and prolateral views. 62. Spermathecae.

NotE: Although no type material exists for this name, only one species of Myrmekiaphila is known from the area of northeastern Alabama including the type locality. Although it would be optimal to designate a male specimen as the neotype, only topotypic females were available.

TyPE: Female neotype, here designated, from Tennessee River, near Scottsboro, Jackson Co., Alabama [34.6371 -85.976, MYR295], 1939, deposited in AMNH. Male exemplar specimen from Ricketts Gap, Bishop Mountain, near Guntersville, S of Hwy 431, Marshall Co., Alabama [34.4551 -86.3417, MYR299], May 5, 1984 (R. Bennett), deposited in AMNH.

Diagnosis: Males can be recognized by the two long, thin palpal prongs, the accessory prong being only slightly shorter and more strongly curved than the embolus (figs. 7, 27), lacking prominent serrations (fig. 27), and by the metatarsal distal dilation being very slender. Females can be distinguished by the laterally originating spermathecal bulbs (tending to appear excessively slanted laterally, fig. 62) and by the posterior sternal sigilla pair forming a distinct depression.

FemAle (NEOTYPE): Specimen preparation and condition. Female collected live from burrow in river bank. Genital plate removed and stored in microvial with specimen. Color. Carapace, legs, chelicerae, dark reddish brown (2.5YR 3/5). Abdomen uniform very dusky red (2.5YR 2.5/2), ventrum and spinnerets pale yellow. Cephalothorax. Carapace 6.56 long, 5.62 wide, glabrous; generally smooth surface, pars cephalica moderately elevated. Fringe lacks setae. Foveal groove deep, slightly procurved. Eye group slightly elevated on low mound. AER slightly procurved, PER slightly recurved, PME, AME subequal in diameter. Sternum moderately setose, STR1 3.76, STRw 3.56. Three pairs of sternal sigilla. anterior pair smaller, oval, marginal; medial pair marginal, moderately elongate; posterior pair much larger, oval, more mesially posi- 
tioned but moderately separated, forming noticeable depression in sternum. Chelicerae, anterior tooth row armed with eight teeth, with large posterior margin denticle patch. Palpal endites with cuspules (ca. 38) concentrated at inner (promargin) posterior heel; labium with six cuspules, LBw 1.36, LB1 0.82 . Rastellum consisting of six very stout spines positioned on mound; fringe of short spines along distal promargin extending upward from rastellum. Abdomen. Moderately setose. Legs. Leg I: 4.70, 2.35, 3.25, 2.20, 1.68; leg IV: $4.95,2.60,3.80,3.24,2.04$. Tarsus I with single staggered row of 12 trichobothria. Leg I with relatively dense scopulae on tarsus and distal half of metatarsus; tarsi II, III with moderately light scopulae (on tarsi only). PT3s numerous (but missing), TB3s numerous (missing). Distinct preening comb on retrolateral distal surface (at tarsus-metatarsus joint) of metatarsi III, IV. Spermathecae. Widely separated, bulbs laterally displaced on elongate base (fig. 62).

Male (exemplar specified above): Specimen preparation and condition. Specimen collected from burrow in ravine bank as juvenile, molted to maturity in captivity, preserved in ca. $70 \%$ ethanol. Coloration presumably faded. Leg I left side and pedipalp right side removed and stored in vial with specimen; pedipalp left side missing. General coloration. Carapace, chelicerae, and legs dark reddish brown (2.5YR 2.5/3). Abdomen uniform reddish black (2.5YR 2.5/1) dorsally, ventrum and spinnerets pale yellow. Cephalothorax. Carapace 5.69 long, 4.94 wide, glabrous, stout black bristles along fringe; surface smooth, pars cephalica elevated. Fringe, posterior margin with black bristles. Foveal groove deep, moderately procurved. Eyes on low mound. AER slightly procurved, PER slightly recurved, PER eyes noticeably smaller in diameter than those of AER. Sternum moderately setose, STR1 3.28, STRw 2.96. Posterior sternal sígilla large, positioned centrally, not contiguous, anterior sigilla pairs small, oval, marginal. Chelicerae with distinct anterior tooth row (seven teeth), posterior margin with single row of small denticles. Palpal endites with patch of small thin cuspules on proximal, inner margin, labium without cuspules, LBw 1.19. LB1
0.61. Rastellum consisting of four very stout spines on mound. Abdomen. Setose, heavier black setae intermingled with fine black setae. Legs. Leg I: 5.25, 2.35, 3.90, 3.00, 2.56; leg IV: $5.25,2.32,4.40,4.08,2,60$. Light tarsal scopulae on legs I-IV. Tarsus I with single, slightly staggered row of ca. nine trichobothria. Leg I spination pattern illustrated in Figures 17, 56-58; TSp 9, TSr 6, TSrd 1. Pedipalp (right side). Articles stout, lacking distinct spines (figs. 7, 59-61). PTw 1.15, PT1 3.00 , Bl 1.29. Ledge on distal-retrolateral surface of palpal tibia, displaced somewhat medially (figs. 59-61). Palpal bulb bearing two thin prongs; auxiliary prong slightly shorter, more curved than embolus, bearing only small serrations (figs. 7, 27).

MATERIAl Examined: UNITED STATES: ALABAMA: Cleburne Co.: Mount Cheaha, Cheaha State Park [33.4857 -85.8091, MYR219], Oct. 28, 1947 (E. Wilson, MCZ), 1 कै . DeKalb Co.: Desoto Resort State Park, main park area $5 \mathrm{mi} \mathrm{S}$ Desoto Falls on County Road 89 [34.5026 -85.6201, MYR224], Sept. 5, 1966 (F. Coyle, AMNH), 4 , spiderlings. Jackson Co.: mound in Tennessee River, near Scottsboro [34.6371 -85.976 MYR222, 223, 295], 1939 (AMNH), 14\%. Marshall Co.: Bishop Mountain, near Guntersville, Ricketts Gap [34.4551 -86.3417, MYR299], Feb. 17, 1984 (R. Bennett, AMNH), 1 है; Hurricane Branch, $1 \mathrm{mi} \mathrm{S}$ Town Creek Bridge, Lake Guntersville State Park, Highway 227 [34,3882 -86.1862, MYR221], Feb. 17, 1984 (R. Bennett, AMNH) 1; Lake Guntersville State Park, $34.40836-86.19834$, elev. $182 \mathrm{~m}$ [MY2019], June 10, 2003 (B. Hendrixson, P. Marek, ECU-AMNH), 1 ; : Little Mountain State Park [34.4462 -86.15, MYR220], July 27, 1966 (F. Coyle, AMNH), 1 ㅇ․

Distribution: Known only from northeastern Alabama (fig. 63).

Myrmekiaphila jenkinsi, new species

Figures $8,18,28,63-70$

Types: Male holotype and female paratype from Fall Creek Falls State Park [35.6715 -85.3666], Van Buren Co., Tennessee (July 24, 1966; F. Coyle), deposited in AMNH. 


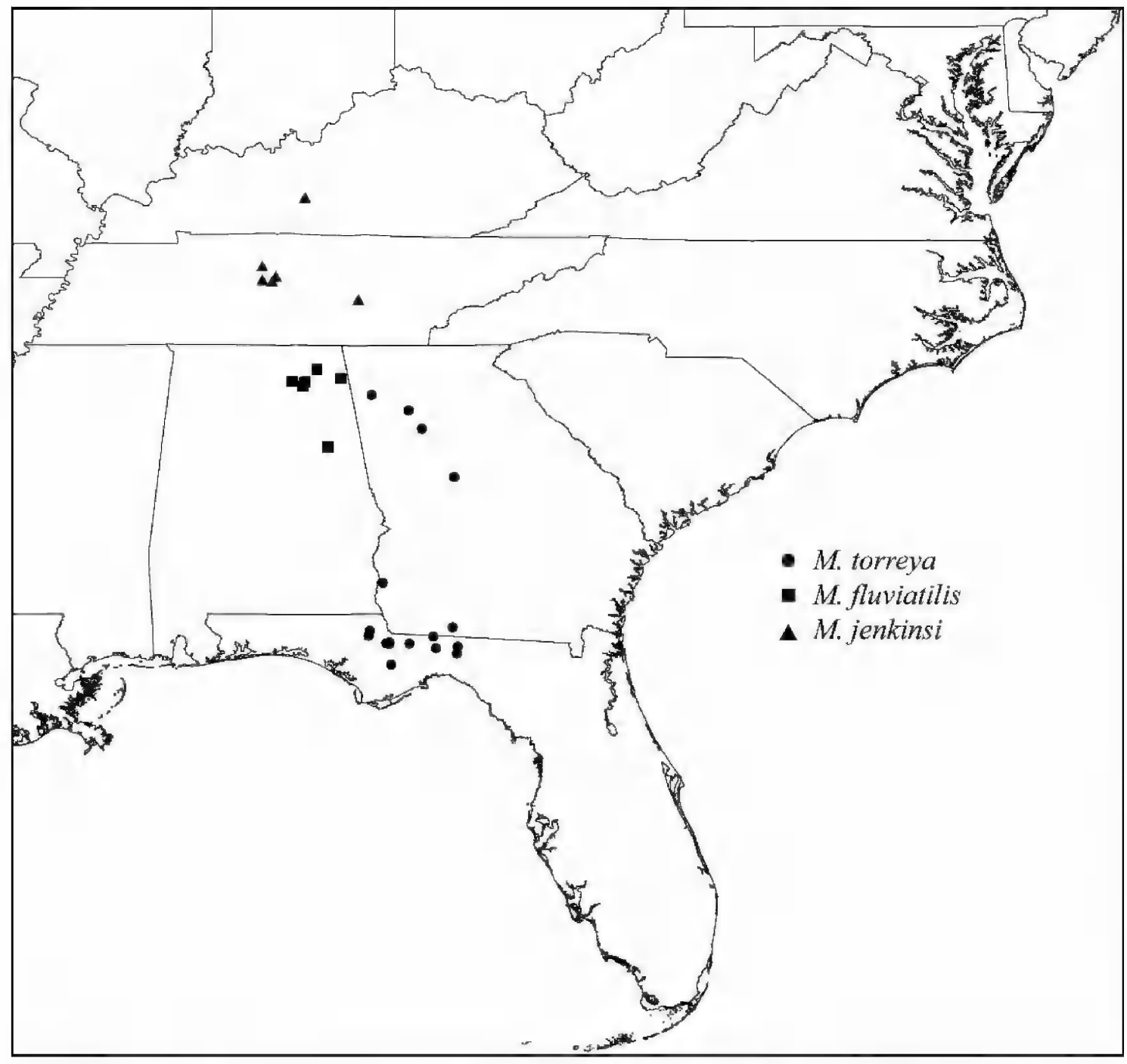

Fig. 63. Records of M. torreya Gertsch and Wallace, M. fluviatilis (Hentz), and M. jenkinsi, new species.

ETYMology: The specific name is a patronym in honor of Dr. R. L. Jenkins of Samford University, Birmingham, Alabama.

Diagnosis: Males can be recognized by an embolus and accessory prong that are subequal in length, the lower accessory prong being only slightly curved (figs. 8,28 ), and by the slender palpal tibia typically having a more proximally situated retrolateral ledge (figs. 8, 69). Females can be distinguished by the laterally originating spermathecal bulbs and more narrow, closely spaced spermathecae (generally lengthened vertically, fig. 70), and by the preening combs being absent on metatarsi III of some specimens.

MALE (HOLOTYPE): Specimen preparation and condition. Specimen collected from burrow, preserved in ca. 70\% ethanol. Coloration appears faded. Pedipalp, leg I left side, leg II right side, removed and stored in vial with specimen. General coloration. Carapace, chelicerae, and legs dark reddish brown (5YR 3/ 4). Abdomen uniform dark brown (7.5YR 3/4) dorsally, ventrum and spinnerets pale yellow, wide dusky stripes on dorsum. Cephalothorax. Carapace 6.56 long, 5.31 wide, glabrous, stout 

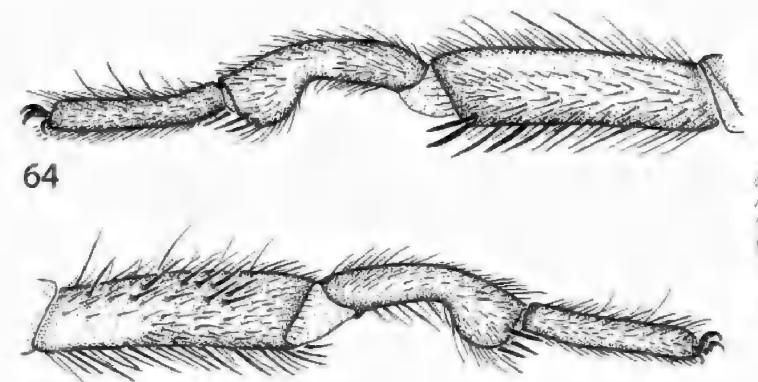

65
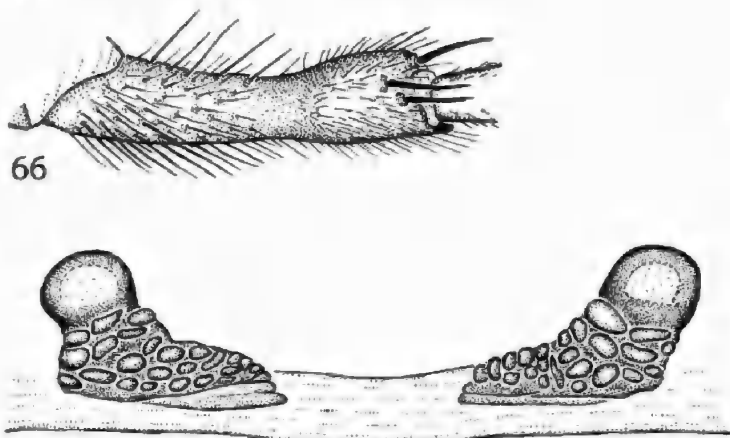

70

Figs. 64-70. Myrmekiaphila jenkinsi, new species, from Davidson Co., Tennessee (male) and Edmonson Co., Kentucky (female). 64-66. Male left leg I: retrolateral, prolateral, and ventral (metarsus) views. 67-69. Male left pedipalp: retrolateral, ventral, and prolateral views. 70. Spermathecae.

black bristles along fringe; surface smooth, pars cephalica elevated. Fringe, posterior margin with black bristles. Foveal groove deep, moderately procurved. Eye group slightly raised, not on tubercle. AER slightly procurved, PER slightly recurved. PME, AME subequal in diameter. Sternum moderately setose, STR1 3.64, STRw 3.28. Posterior sternal sigilla large, positioned centrally, not contiguous, anterior sigilla pairs small, oval, marginal. Chelicerae with distinct anterior tooth row (10 teeth), posterior margin with patch of small denticles. Palpal endites with patch of small, thin cuspules on proximal, inner margin, labium with single cuspule, LBw 1.17, LBl 0.67. Rastellum consisting of four or five very stout spines on mound. Abdomen. Setose, heavier black setae intermingled with fine black setae. Legs. Leg I: 5.50, 2.60, 4.40, $3.20,2.88$; leg IV: $5.62,2.40,4.72,4.64,3.20$. Light tarsal scopulae on legs I, II. Tarsus I with single, slightly staggered row of seven trichobothria. Leg I spination pattern illustrated in Figures 18, 64 66; TSp 8, TSr 13, TSrd 1. Pedipalp. Articles stout, lacking
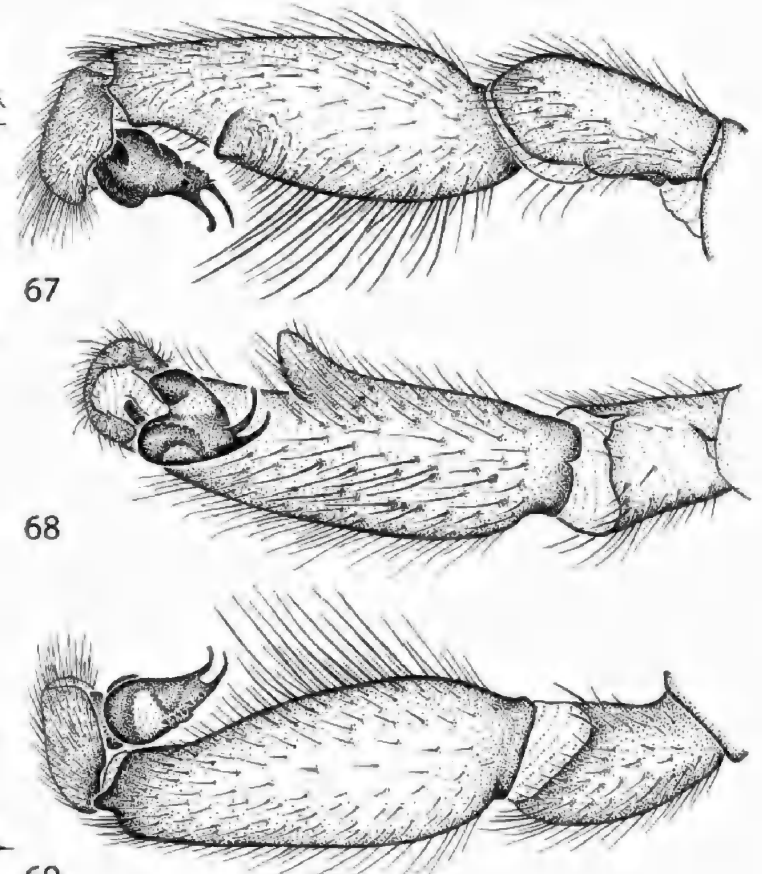

69 distinct spines (figs. 8, 67-69). PTw 1.28, PTl $3.20, \mathrm{Bl} 1.25$. Ledge on distal-retrolateral surface of palpal tibia, positioned at proximal two-thirds of length. Palpal bulb (fig. 28) distally bifurcate, embolus relatively slender, auxiliary prong slender, subequal in length to embolus, small serrations proximal.

Variation (3). Cl 6.50-6.93, Cw 5.31-5.43; STRl 3.40-3.64, STRw 3.28-3.36; LBw 1.101.42 , LBl $0.67-0.75$; leg I: $5.50-5.75,2.50-$ $2.60,4.16-4.40,3.16-3.20,2.68-2.88$; leg IV: $5.00-5.68,2.20-2.44,4.20-4.72,4.40-4.64$, 2.80-3.20; PTw 1.25-1.40, PT1 3.20-3.40, Bl 1.22-1.30; TSp 8-11, TSr 7-13.

Female (PARATYPE): Specimen preparation and condition. Female collected from burrow, preserved with male holotype. Genital plate removed and stored in microvial with specimen. Color. Carapace, legs, chelicerae, yellowish red (5YR 4/6). Abdomen uniform dark reddish brown dorsally ( $5 \mathrm{YR} 3 / 3$ ), ventrum and spinnerets pale yellow; recently collected specimens have wide dusky stripes on dorsum. Cephalothorax. Carapace 6.56 long, 5.56 wide, glabrous; generally smooth surface, pars 
cephalica moderately elevated. Fringe lacks setae. Foveal groove deep, procurved. Eye group slightly elevated on low mound. AER slightly procurved, PER slightly recurved. PME, AME subequal in diameter. Sternum moderately setose, STRI 3.84, STRw 3.64. Three pairs of sternal sigilla, anterior pairs small, oval, marginal, posterior pair much larger, oval, more mesially positioned. Chelicerae, anterior tooth row armed with nine teeth, posterior margin with denticle patch. Palpal endites with cuspules (ca. 27) concentrated at inner (promargin) posterior heel; labium with one cuspule, $\mathrm{LBw} 1.47, \mathrm{LB} 1$ 0.87 . Rastellum consisting of three very stout spines positioned on mound; fringe of short spines along distal promargin extending upward from rastellum. Abdomen. Moderately setose. Legs. Leg I: 4.56, 2.40, 3.16, 2.16, 1.60; leg IV: $4.44,2.48,3.60,2.88,1.80$. Tarsus I with single staggered row of seven trichobothria. Legs I-IV with moderately light scopulae on tarsi, light scopulae on distal third of metatarsus. PT3s 14, TB3s-dense patch of thin spines. Distinct preening comb on retrolateral distal surface (at tarsus-metatarsus joint) of metatarsus IV, absent on metatarsus III. Spermathecae. Two simple spermathecal bulbs, arranged in transverse row, consisting of moderately sclerotized stalk, somewhat laterally positioned, and enlarged, lightly sclerotized apical bulb (fig. 70).

Variation (3). Cl 6.56-7.50, Cw 5.56-6.18; STRI 3.84 4.16, STRw 3.64-3.96; LBw 1.371.50, LBI $0.87-1.00$; leg I: $4.56-5.25,2.40$ $2.76,3.16-3.48,2.16-2.40,1.60-1.80$; leg IV: $4.44-5.25,2.48-2.80,3.60-4.06,2.88-3.60$, $1.80-2.28$.

MATERIAL EXAMINED: UNITED STATES: KENTUCKY: Edmonson Co: Lincoln Trailhead, ca $0.5 \mathrm{mi}$ SW KY-1827 on Ollie Road, 37.25555 -86.15842 [MY2836], May 5, 2004 (B. Hendrixson, ECU-AMNH), 1 ; Mammoth Cave National Park [37.1834 -86.15, MYR 199], Sept. 16, 1966 (F. Coyle, AMNH), $1 \mathcal{1}$, spiderlings. TENNESSEE: Davidson Co.: Nashville [36.1659-86.7844, MYR 151], Nov. 10, 1958 (A. Laskey, AMNH), 18 ; Nashville [36.1659-86.7844, MYR153], Aug. 1942 (R. Wheeler, AMNH), 2․ Rutherford Co.: La Vergne [36.0156 -86.5819, MYR152], Oct. 20, 1936 (C, Crosby, CUC), 1 . 1 juv. Van Buren
Co.: Fall Creek Falls State Park [35.6715 -85.3566, MYR155], July 24, 1966 (F. Coyle, $\mathrm{AMNH}), 1 \delta, 1+$. Williamson Co.: Brentwood, 1 mi E I-65 on Moore's Lane [35.9646 -86.7883, MYR303], June 3, 1998 (L. Roy, AMNH), 1 ; ; Nolensville, York Road [35.937 -86.642, MYR 154], Dec. 1, 1996 (R. Jenkins, AMNH), 1 tै

Distribution: Western Kentucky south to Tennessee (fig. 63).

Myrmekiaphila torreya Gertsch and Wallace Figures 9, 19, 29, 63, 71-77

Myrmekiaphila torreva Gertsch and Wallace, 1936: 15, figs. 15, 17-25 (male holotype and female allotype from Torreya Ravine, Liberty Co., Florida, in AMNH, examined).

Diagnosis: Males can be recognized by the presence of a relatively slender palpal prong and embolus, with the shorter auxiliary prong subequal in diameter to the embolus (figs. 9, 29). Females are difficult to definitively diagnose, but have spermathecae with an enlarged base that extends subequally from a central bulb that lacks an elongate stalk (fig. 77).

Descriptions: Male and female described by Gertsch and Wallace (1936).

MATERIAl EXAMINED: UNITED STATES: FLORIDA: Gadsden Co.: no specific locality [30,5633 84.6027, MYR211], Apr. 14, 1935 (H. Wallace, AMNH), 1 ㅇ․ Jackson Co.: Merritts Mill Pond, $3.5 \mathrm{mi} E$ Marianna on U.S. 90 [30.753 -85.1933, MYR215], Oct. 9, 1967 (F. Moore, FSCA), 1 ; 7 mi S Marianna on State Road 71, in woods, 100 yards from Chipola River [30.6815 -85.2069, MYR277], Aug. 4, 1963 (B. Mansell, F. Moore, AMNH), 1\%, spiderlings. Jefferson Co.: no specific locality [30.4169 -83.8957, MYR241], Apr. 7. 1969 (H. Wallace, FSCA), 28; Big Bend Horticulture Lab [30.516 -83.888 MYR106108], Apr. 7-28, 1969 (FSCA), $3 \hat{\delta}$. Leon Co.: no specific locality [30.4946 -84.2082, MYR210, 263, 264], Apr. 15-16, 1936 (R. Bellamy, J. Rogers, H. Wallace, FSCA), $8 \stackrel{9}{+} 4$ juv.; Tallahassee, Tall Timbers Research Station [30.658769-84.208445, MYR109$114,227,229-235,238,239,246,258-260]$, Mar.-June 1970 (D. Harris, W. Whitcomb, FSCA), 36 है, 2ㅇ, 2 juv., [MYR258], Apr. 9, 

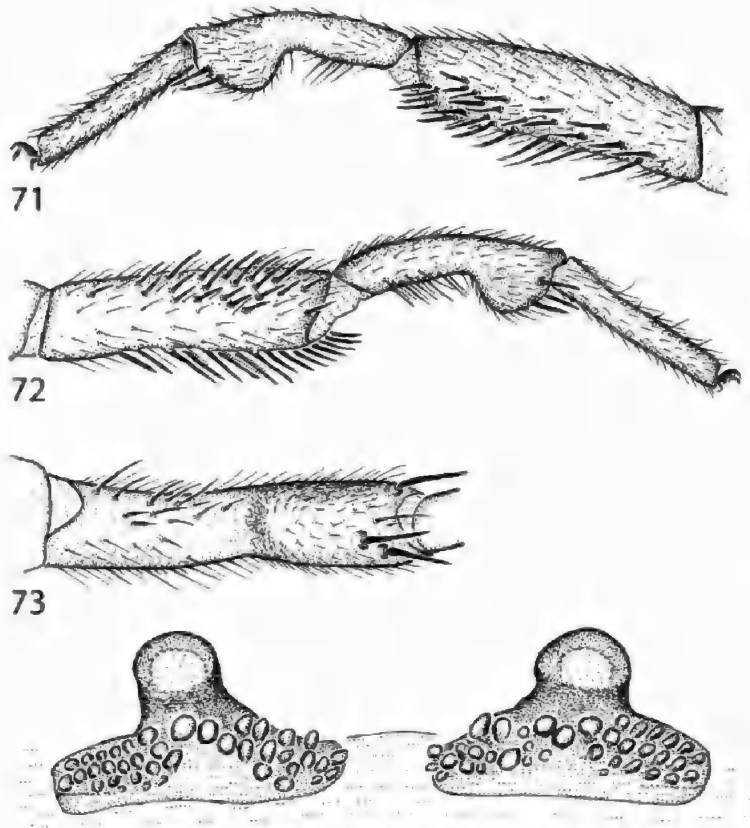

77
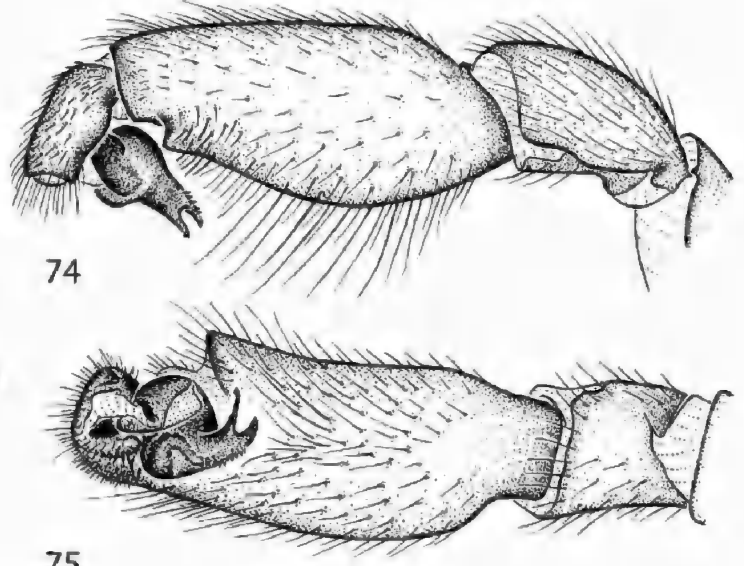

75

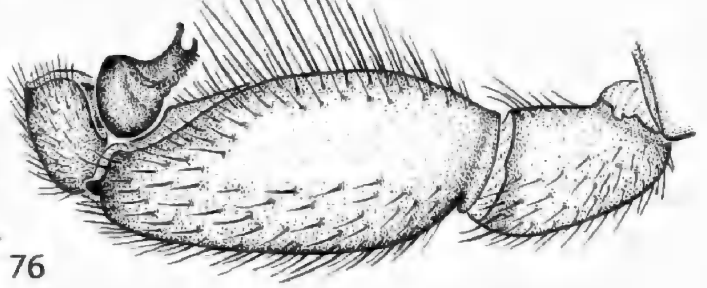

Figs, 71-77. Myrmekiaphila torreyi Gertsch and Wallace from Liberty Co., Florida, 71-73. Male left leg 1: retrolateral, prolateral, and ventral (metarsus) views. 74-76. Male left pedipalp: retrolateral, ventral, and prolateral views. 77. Spermathecae.

1970 (R. Woodruff, FSCA), 1ㅇ, [MYR259, 260], Aug-Sept. 1968 (W. Baker, FSCA), 19 , 1 juv. Liberty Co.: no specific locality [30.2508 -84.8755, MYR205], Nov. 4, 1938 (H. Wallace, FSCA), 1, [MYR213], Apr. 8, 1941 (H. Wallace, FSCA), 1 \%, [MYR206, 207], Apr. 14, 1951 (H, Wallace, FSCA), 2 . 2 juv., [MYR242], June 4, 1953 (H. Wallace, FSCA), 1\%, [MYR216], Aug. 18, 1960 (JMCC, MCZ), 8우 just E Apalachicola on FL-20, 30.43181 -84.33987 [MY2567-2572], Feb. 27, 2004 (B. Hendrixson, D. Beamer, ECU-AMNH), 6 ; near Sweet Water in deep ravine, $30.51075-84.95982$, elev. $73 \mathrm{~m}$ [MY2575, 2576], Feb. 28, 2004 (B. Hendrixson, D. Beamer, ECU-AMNH), 2 ; Torreya Ravine, Torreya State Park, $10 \mathrm{mi}$ NE Bristol, 30.56678 -84.95, [MYR208], Apr. 10, 1935, (H. Wallace, FSCA), 2 \%, [MYR237, 270, 273], Apr. 10, 1935 (H. Wallace, AMNH), 4 , one with spiderlings, [MYR217, 218, 240, 262, 272, 276], Apr. 1718, 1936 (R. Bellamy, H. Wallace, AMNH, MCZ), 50 \& , 16 \% , 6 juv., [MYR275], Apr. 16, 1938, (W. Gertsch, AMNH), 6\%, [MYR148],
Apr. 8, 1941 (H. Wallace, FSCA), 1 , [MYR102], June 4, 1953 (H. Wallace, FSCA), 1․, [MYR261], Mar. 10, 1963 (AMNH), 1 juv., [MYR274], Apr, 2, 1963 (W. Shear, AMNH), 2q, [MYR104], Apr. 20, 1963 (A. Derbonne, FSCA), 1\%, [MYR236], Mar. 15, 1964 (F. Moore, FSCA), 1 oै . [MYR115], Mar. 29, 1964 (AMNH), 1 §ै, Mar. 31, 1964 (A. Weaver, F. Coyle, VMNH), 1 \&, [MYR 197], Mar. 31, 1965 (J. Beatty, JAB), 2 +, [MYR265269, 271], May 1965, Apr., Sept. 1967 (F. Moore, FSCA), 11\%, 2 juv., Aug. 16, 1966 (W. Shear, VMNH), 2 + , [MYR 105], May 18, 1973 (G. Edwards, FSCA), 1 \%, [MYR103], Mar. 10, 1978 (D. Richman, FSCA), 2\%; [MYR226], Mar. 31, 1979 (P. Choate, FSCA), 28, [MYR286], Feb. 15, 1986 (D. Folkerts, AMNH), 1 . GEORGIA: Cob Co.: Kennesaw, Chastain Lakes [34.0234 -84.6155, MYR083], Nov. 15, 1997 (H. McNatt, AMNH), 18 . Early Co.: $8.5 \mathrm{mi}$ NNW Blakely [31.4645 -84.99667, MYR001-003], Mar. 24, 1992 (G. Folkerts, AMNH), 3 \% . Floyd Co.: Rome [34.257 -85.1647, MYR248], (R. Battey, MCZ), 1\%. Fulton Co.: Atlanta 

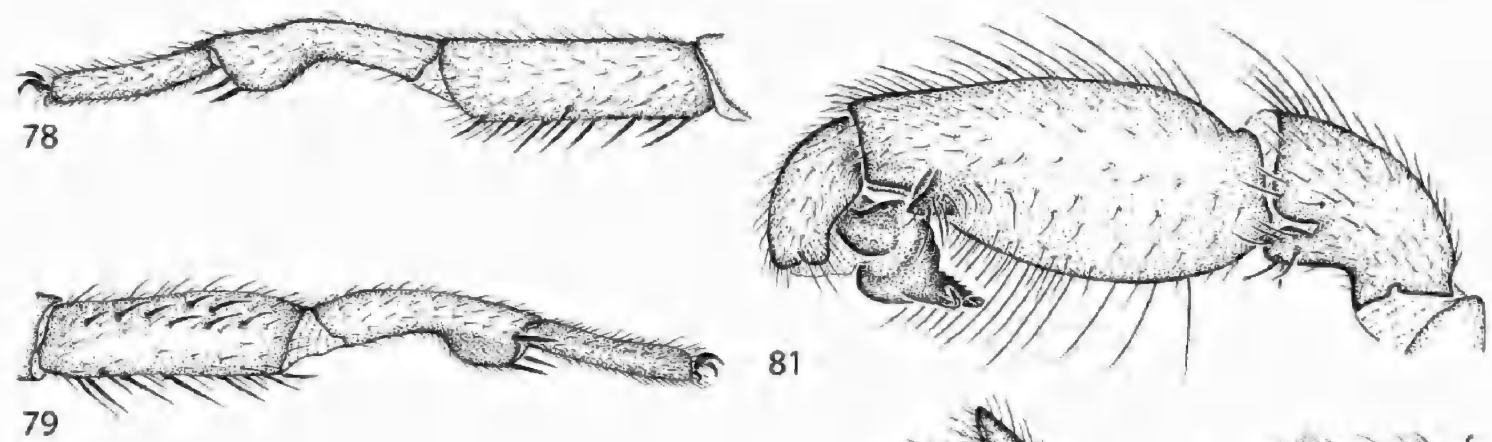

81

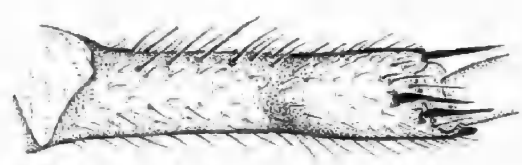

80

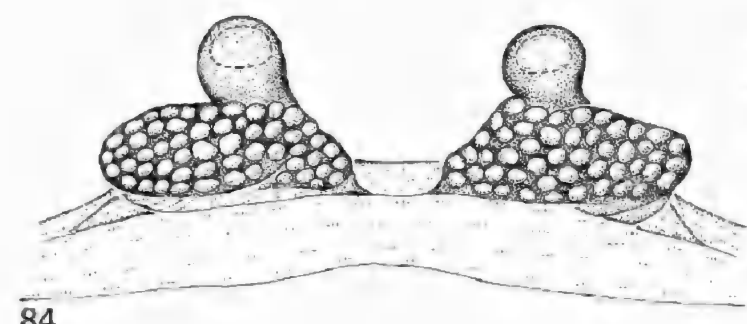

84
82
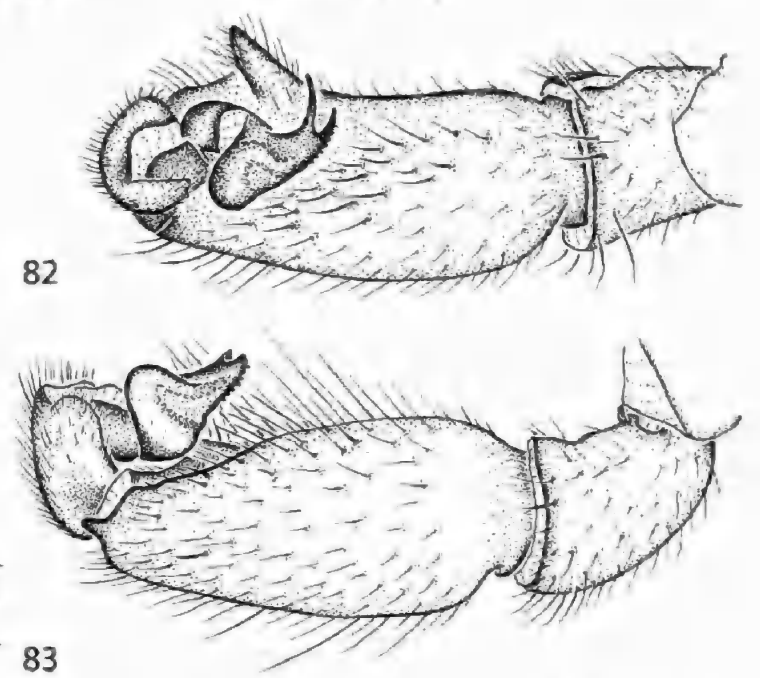

Figs. 78-84. Myrmekiaphila neilyoungi, new species, from Tuscaloosa Co., Alabama. 78-80. Male left leg I: retrolateral, prolateral, and ventral (metarsus) views. 81-83. Male left pedipalp: retrolateral, ventral, and prolateral views. 84. Spermathecae.

[33.75 -84.41667, MYR203, 250], Apr. 14-16, 1946 (P. Fattig, AMNH), 1 fै, 1 9. Monroe Co.: Forsyth [33.03438 -83.9382, MYR209], Nov. 1, 1963 (N. Mason, AMNH), 1 \%. Thomas Co.: SE edge Thomasville, Millpond Plantation [30.807 -83.9627, MYR212], Mar. 31, 1968 (W. Sedgwick, AMNH), 1 , , spiderlings, [MYR214], June 16, 1973 (F. Coyle, W. Shear, AMNH), 1 , spiderlings.

Distribution: Known only from Georgia and adjacent Florida (fig. 63).

\section{Myrmekiaphila neilyoungi, new species}

Figures 2, 3, 10, 20, 30, 78-85

TYPES: Male holotype and female paratype from Samford University Campus [33.46299 -86.76222], Jefferson Co., Alabama (April 30, 1998; J. Powell), deposited in AMNH.
Eтrmology: The specific name is a patronym in honor of Mr. Neil Young, in recognition of his social activism for peace over the course of his distinguished musical career.

Diagnosis: Males can be recognized by the presence of an elongate, well-defined ledge on the palpal tibia, which is excavated retrolaterally (best seen in ventral view), the long embolus and much shorter auxiliary prong (with its tip usually thick but lacking a distinct ledge, figs. 10, 30, 81-83). Females, difficult to distinguish morphologically, have spermathecae with an enlarged basal region, and bulbs originating from the base proximal to the central margin (fig. 84).

MALE (HOLOTYPE): Specimen preparation and condition. Specimen preserved in ca. 70\% ethanol. Coloration appears to be faded. Pedipalp, leg I left side removed and stored in vial with specimen. General coloration. Carapace, chelicerae, and legs dark_reddish 


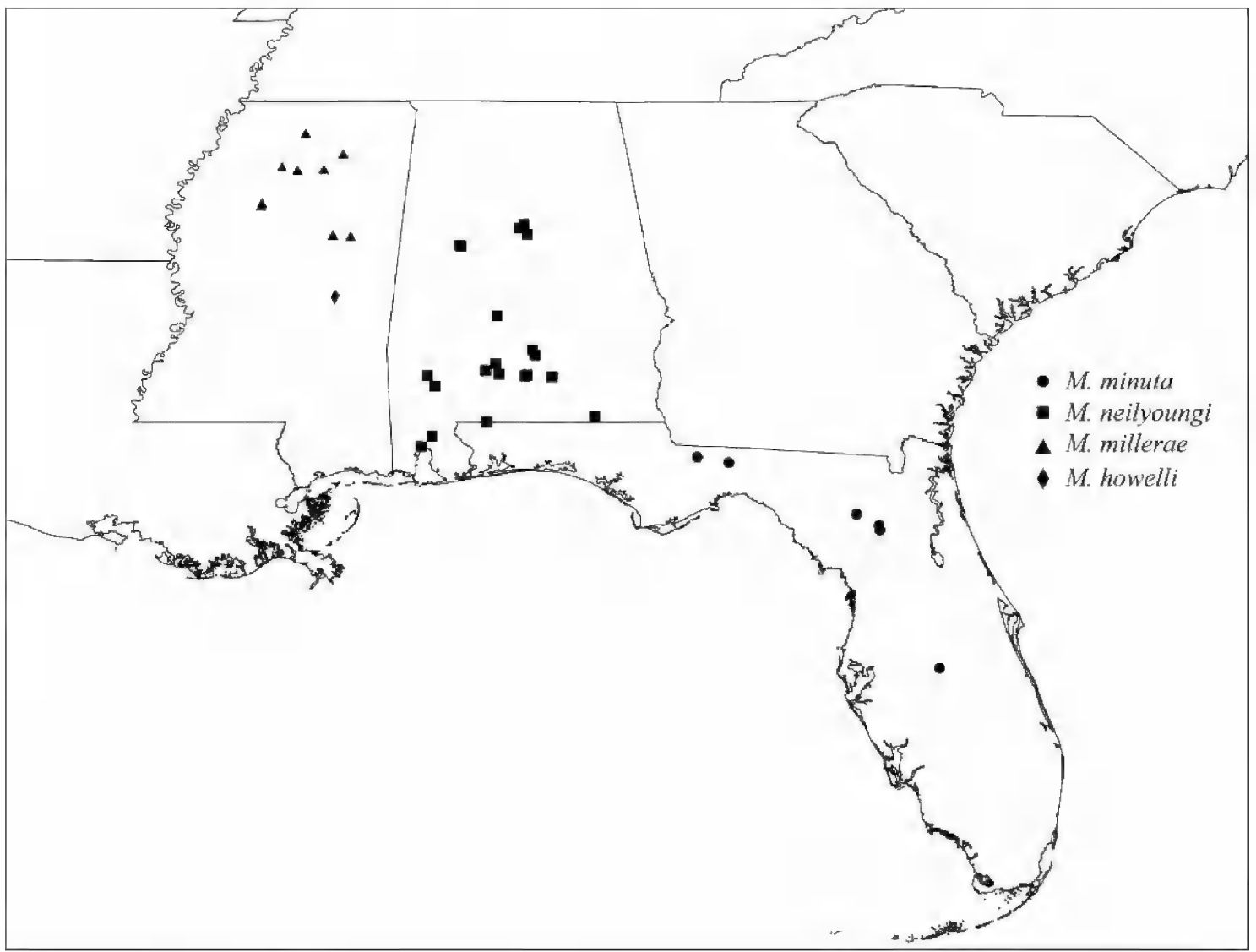

Fig. 85. Records of M. minuta, new species, $M$. neilyoungi, new species, $M$. millerae, new species, and $M$. howelli, new species.

brown (2.5YR 2.5/3). Abdomen uniform dark brown (2.5YR 2.5/1) dorsally, ventrum and spinnerets pale yellow, dorsum lacks faint stripes observed in other specimens. Cephalothorax. Carapace 7.19 long, 5.75 wide, glabrous, stout black bristles along fringe; surface smooth, pars cephalica elevated. Fringe, posterior margin with black bristles. Foveal groove deep, straight to slightly recurved. Eye group slightly raised, not on tubercle. AER slightly procurved, PER slightly recurved. PME, AME subequal in diameter. Sternum moderately setose, STR1 3.92, STRw 3.56. Posterior sternal sigilla very large, positioned centrally, not contiguous, anterior sigilla pairs small, oval, marginal. Chelicerae with distinct anterior tooth row (eight teeth), posterior margin with patch of small denticles. Palpal endites with patch of small cuspules on proximal, inner margin, labium lacking cus- pules, LBw 1.10, LB1 0.68. Rastellum consisting of five very stout spines on small mound. Abdomen. Setose, heavier black setae intermingled with fine black setae. Legs. Leg I: 5.62, 2.60, 4.08, 3.16, 2.60; leg IV: 5.44, 2.60, $4.68,4.80,2.88$. Light tarsal scopulae on legs I, II. Tarsus I with single, staggered row of 11 trichobothria. Leg I spination pattern illustrated in Figures 20, 78-80; TSp 4, TSr 9, TSrd 1. Pedipalp. Articles stout, lacking distinct spines (figs. 10, 81-83). PTw 1.55, PT1 3.00, Bl 1.14. Elongate ledge on distalretrolateral surface of palpal tibia, positioned distally. Palpal bulb (fig. 30) distally bifurcate, embolus relatively slender, auxiliary prong thick, shorter in length than embolus, with distinct serrations.

Variation (3). Cl 7.19-8.00, CW 5.75-6.25; STR1 3.92-4.32, STRw 3.52-3.80; LBw 1.101.22 , LB1 $0.68-0.82$; leg I: $5.50-5.81,2.60-$ 
$3.00,4.04-4.16,3.16-3.40,2.60-2.88$; leg IV: $5.44-6.19,2.60,4.68-5.00,3.24-4.80,2.72-$ 3.00; PTw 1.47-1.55, PT1 3.00, Bl 1.05-1.14; TSp 4-5, TSr 9-10.

Female (PARATYPe): Specimen preparation and condition. Female collected live, preserved with male holotype. Genital plate removed and stored in microvial with specimens. Color. Carapace, legs, chelicerae, dark reddish brown (5YR 3/4). Abdomen uniform dark brown dorsally (7.5YR 3/2), ventrum and spinnerets pale yellow; recently collected specimens have wide dusky stripes on dorsal surface of abdomen. Cephalothorax. Carapace 7.76 long, 6.50 wide, glabrous, with generally smooth surface, pars cephalica moderately elevated. Fringe lacks setae. Foveal groove deep, procurved. Eye group slightly elevated on low mound. AER slightly procurved, PER slightly recurved, PME, AME subequal in diameter. Sternum moderately setose, STR1 4.36, STRw 3.92. Three pairs of sternal sigilla, anterior pairs small, oval, marginal, posterior pair much larger, oval, more mesially positioned. Chelicerae, anterior tooth row armed with 12 teeth, posterior margin with denticle patch. Palpal endites with numerous cuspules $(>30)$ concentrated at inner (promargin) posterior heel; labium with four cuspules, LBw 1.53, LB1 0.97 . Rastellum consisting of five very stout spines positioned on mound; fringe of short spines along distal promargin extending upward from rastellum. Abdomen. Moderately setose. Legs. Leg I: 5.31, 2.80, 3.60, 2.60, 1.80; leg IV: $5.44,3.12,4.25,3.75,2.19$. Tarsus I with single staggered row of eight trichobothria. Legs I-IV with moderately light scopulae on tarsi, light scopulae on distal one-third of metatarsi I, II. PT3s $>20$, TB3s-dense patch of thin spines. Distinct preening comb on retrolateral distal surface (at tarsus-metatarsus joint) of metatarsi III, IV. Spermathecae. Two simple bulbs, arranged in transverse row, consisting of moderately sclerotized stalk and enlarged apical bulb, lightly sclerotized, borne medially on enlarged base (fig. 84).

Variation (3). Cl 6.25-7.76, Cw 5.31-6.81; STR1 3.48-4.76, STRw 3.24 4.00; LBw 1.221.53, LBI $0.82-0.97$; leg I: 4.00-5.75, 2.40$2.80,2.80-3.80,2.20-2.60,1.40-1.80$; leg IV: $4.12-5.44, \quad 2.36-3.12,3.60-4.44,3.12-3.81$, $1.80-2.19$.
MATERIAl ExAmined: UNITED STATES: ALABAMA: Butler Co.: no specific locality [31.8293 -86.6208, MYR188], Apr. 1, 1967 (R. Hamilton, AMNH), 19; Fort Dale Cemetery, $4.5 \mathrm{mi} \mathrm{N}$ Greenville [31.89549 -86.655, MYR255], Apr. 12, 1940 (A. Archer, AMNH), $1 \hat{\delta} ; 3 \mathrm{mi}$ NW McKenzie on US Route 31 [31.5858 -86.717, MYR096], June 6, 1962 (K. Brandon, AMNH), 4, [MYR091], Apr. 5, 1963 (J. MacMahon, AMNH), 5, [MYR093], Mar. 20, 1967 (B. Valentine, AMNH), 4ㅇ, [MYR098], Apr. 6, 1968 (J. Beatty, JAB), 12 \&, 6 juv., [MYR099], June 21, 1973 (R. Little, CAU), 2 ; ; ca $1.2 \mathrm{mi} \mathrm{NW}$ junction AL-55 on US-31, just NW McKenzie, 31.56635 -86.74021, elev. $93 \mathrm{~m}$ [MY25372540], Feb. 26, 2004 (B. Hendrixson, D. Beamer, ECU-AMNH), 4\%; Persimmon Creek, 3 mi N Mckenzie [31.57559 -86.7401, MYR094], June 17, 1967 (N. Rubenstein, AMNH), 3 ㅇ. Baldwin Co.: Hurricane Landing, Mobile-Tensaw Delta WMA, 30.81922 -87.91383, elev. 20 m [MY2548], Feb. 26, 2004 (B. Hendrixson, D. Beamer, ECU-AMNH), 19. Clarke Co.: $5 \mathrm{mi} S$ Jackson [31.446 -87.8717, MYR085], Mar. 20, 1998 (H. McNatt, AMNH), 1 ; 2 mi NW Jackson, Little Stave Creek [31.5496 -87.93, MYR097], June 1, 1963 (R. Brandon, AMNH), 2 \% , 1 juv.; near Jackson Creek on AL-69, 31.59195 -87.97807, elev. 33 m [MY2035-2036], June 14, 2003 (B. Hendrixson, P. Marek, ECUAMNH), 2ㅇ․ Connecuh Co.: $10.7 \mathrm{mi} \mathrm{N}$ Cane creek on Route 83, small tributary of West Sepulga River [31.5968 -87.0706, MYR142], May 10, 1963 (R. Brandon, AMNH), 2 \% . Crenshaw Co.: $0.8 \mathrm{mi} \mathrm{N}$ Leon on County Road 77 [31.5677 -86.4013, MYR090, 280283], Mar. 2, 1986 (G. Folkerts, CDF), 4웅 $1 \mathrm{mi} \mathrm{W}$ Leon on County Road 37 [31.5576 -86.4095, MYR278, 279], Mar. 2, 1986 (G. Folkerts, CDF), 2 . Dallas Co.: Cahaba river at Overton Road [32.32316 -87.10021, MYR082], Feb. 24, 1997 (M. Bell, R. Jenkins, AMNH), 1 \% . Geneva Co.: Goose Hollow Road [31.0688-85.878, MYR304], Feb. 15, 1997 (W. Howell, D. Coffey, C. Patrick, AMNH), 2 . Jefferson Co.: Samford University Campus [33.46299-86.76222], [MYR095], Apr. 16, 1998 (H. McNatt, A. Duke, AMNH), 1 శ。, [MYR080], Apr. 17, 1998 (A. Duke, AMNH), 18, [MYR058], Apr. 30, 1998 (J. Powell, 
AMNH), 1f , 1q; Birmingham, corner of VanBuren and Tyler Roads [33.4155 -86.815, MYR081], Feb. 6, 1997 (R. Jenkins, W. Howell, AMNH), 2q. Mobile Co.: Mobile City Limits [30.6944 -88.0431, MYR302], Apr. 4, 2004 (R. Jenkins, W. Howell, AMNH), 1 \% . Monroe Co.: $5.2 \mathrm{mi}$ W Midway on State Road 47 [31.7262 -87.1128, MYR092, 285, 287], Feb. 22, 1986 (D., G. Folkerts, CDF), 3; $\mathrm{N}$ Tunnel Springs [31.6427 -87.24, MYR089], Jan. 23, 1969 (T. Schwarmer, CAU), 1 ․ Shelby Co: Oak Mountain State Park [33.3429 -86.7211, MYR079], Apr. 30, 1997 (M. Bell, R. Jenkins, AMNH), 19, [MYR256], Sept. 22, 1997 (R. Jenkins, W. Howell, AMNH), $1 \delta$. Tuscaloosa Co.: Tuscaloosa [33.2098 -87.5692, MYR196], Feb. 8, 1951 (C. Hollis, AMNH), 18; University of Alabama [33.1934 -87.54696, MYR297], Apr. 21, 1970 (K. Manuel, CAU), 1 \%ै, [MYR198], (AMNH), 1․ FLORIDA: Santa Rosa Co.: just E Escambia River on FL4, 30.95616 -87.21464, elev. 16 m [MY25512555], Feb. 27, 2004 (B. Hendrixson, D. Beamer, ECU-AMNH), 1 \&े, 3 q, 1 juv.

DistribUTION: Widely distributed throughout Alabama, plus one known locality in northwestern Florida (fig. 85).

\section{Myrmekiaphila millerae, new species}

Figures 11, 21, 31, 85-91

TyPES: Male holotype, three male paratypes, and female paratype from T22N, R3E, Sec. 31NW, Grenada Co., Mississippi (July 10-16, 1991, and Mar. 3-Apr. 13, 1992; G. Baker, P. Miller, G. Snodgrass), deposited in AMNH.

Etymology: The specific name is a patronym in honor of Dr. Patricia Miller.

Diagnosis: Males can be recognized by the presence of a short, stubby ledge on the palpal tibia, a tibia without a noticeable retrolateral excavation in ventral view, and a long embolus and much shorter auxiliary prong, with its tip thick and bent toward the embolus (figs. 11, 31). As with other species in the fluviatilis group, females are difficult to diagnosis due to the paucity of morphological differences, but the spermathecal bulbs appear to have more elongate stalks and smaller bases than those of the other species (fig. 92), and have the posterior pair of sternal sigilla nearly contiguous.

Male (holotype): Specimen preparation and condition. Specimen collected live in pitfall trap, preserved in ca. 70\% ethanol. Coloration appears to be faded. Pedipalp, leg I left side, removed and stored in vial with specimen. General coloration. Carapace, chelicerae, and legs dark reddish brown (2.5YR 3/6). Abdomen uniform dark brown (5YR 4/3) dorsally, ventrum and spinnerets pale yellow, wide dusky stripes on dorsum. Cephalothorax. Carapace 5.62 long, 4.50 wide, glabrous, stout black bristles along fringe; surface smooth, pars cephalica elevated. Fringe, posterior margin with black bristles. Foveal groove deep, slightly procurved. Eye group slightly raised, not on tubercle. AER slightly procurved, PER slightly recurved, PME, AME subequal in diameter. Sternum moderately setose, STR1 3.00, STRw 2.80. Posterior sternal sigilla very large, positioned centrally, not contiguous, anterior sigilla pairs small, oval, marginal. Chelicerae with distinct anterior tooth row (11 teeth), posterior margin with patch of small denticles. Palpal endites with patch of regularly shaped cuspules on proximal, inner margin, labium with four cuspules, LBw 1.00, LBl 0.65. Rastellum consisting of two very stout spines on small mound. Abdomen. Setose, heavier black setae intermingled with fine black setae. Legs. Leg I: 4.81, 2.16, 3.44, 2.76, 2.36; leg IV: 4.75, 2.44, $4.00,3.88,2.68$. Light tarsal scopulae on legs I, II. Tarsus I with single, slightly staggered row of nine trichobothria. Leg I spination pattern illustrated in Figures 21, 86-88; TSp 8, TSr 16, TSrd 1. Pedipalp. Articles stout, lacking distinct spines (figs. 11, 89-91). PTw 1.30, PT1 2.37, B1 0.92. Ledge on distal-retrolateral surface of palpal tibia prominent, positioned distally (figs. 89, 90). Palpal bulb (fig. 31) distally bifurcate, embolus relatively slender, auxiliary prong thick, much shorter in length than embolus, with distinct bend toward embolus.

Variation (3). Cl 5.62-5.93, Cw 4.50-4.87; STR1 3.00-3.24, STRw 2.80-3.00; LBw 1.00, LB1 0.62-0.5; leg I: 4.81-5.18, 2.16-2.36, 3.44$3.80,2.60-2.80,2.36-2.40$; leg IV: 4.69-4.87, $2.20-2.44, \quad 3.60-4.00, \quad 3.844 .00, \quad 2.40-2.68$; 

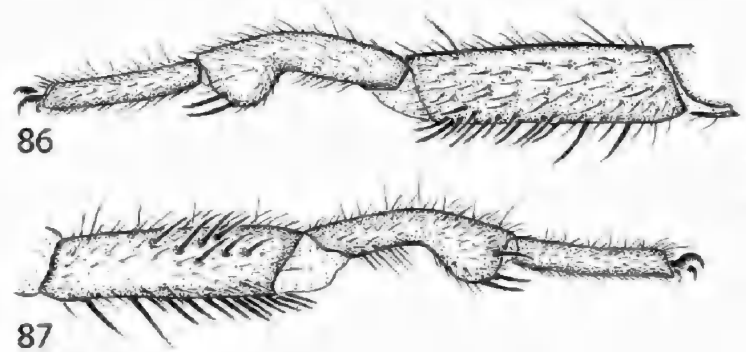

87

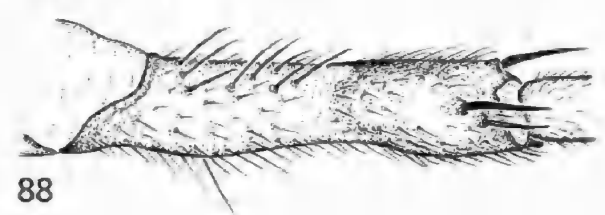

89
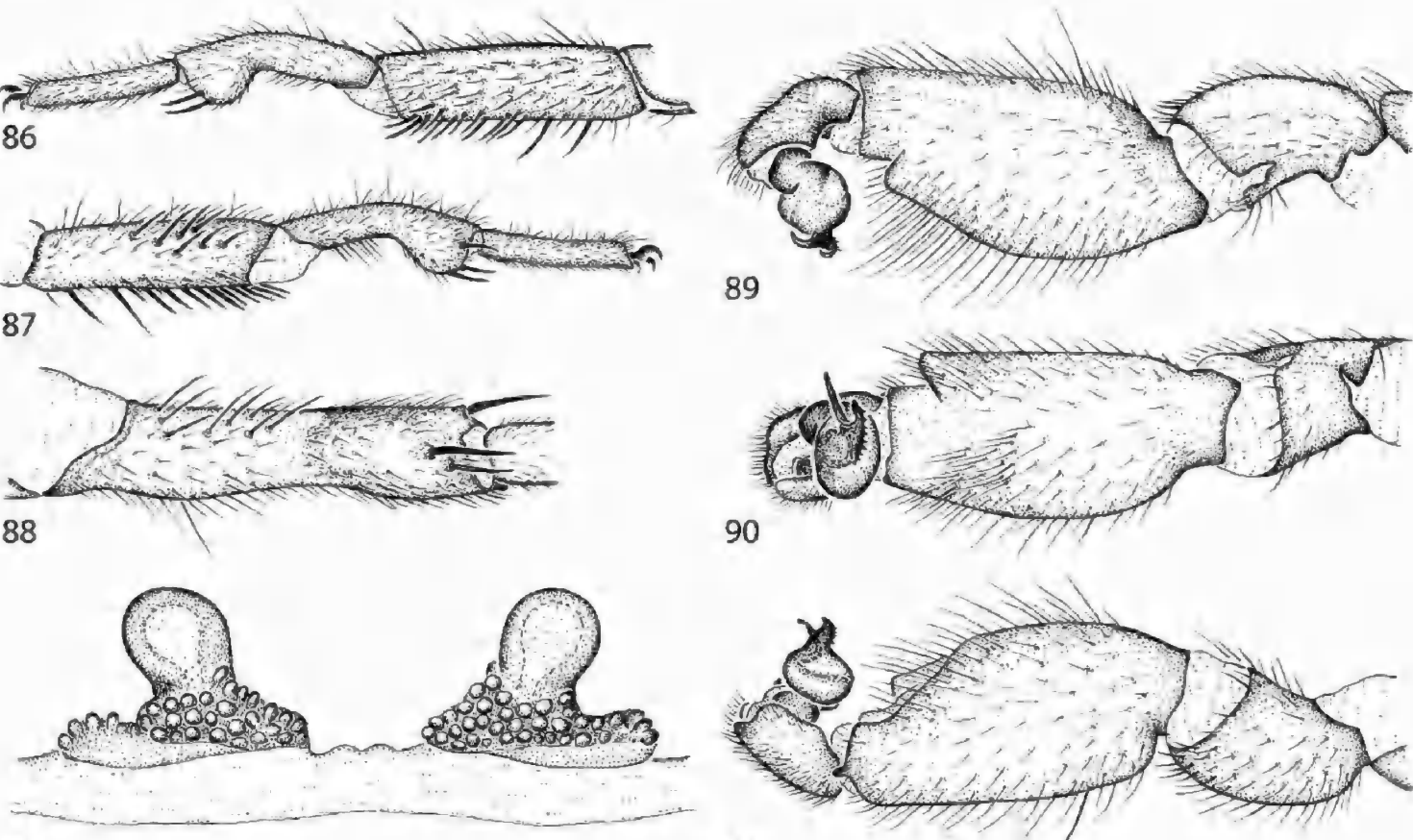

92

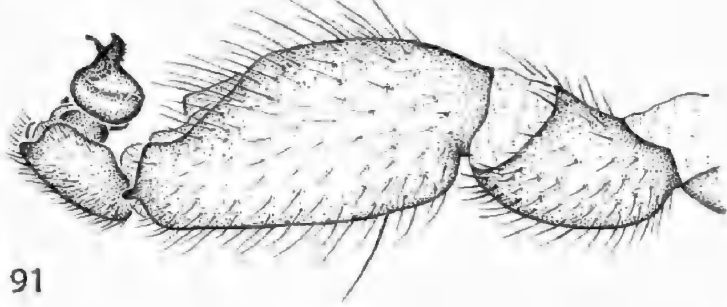

Figs. 86-92. Myrmekiaphila millerae, new species, from Grenada Co., Mississippi. 86-88. Male left leg I: retrolateral, prolateral, and ventral (metarsus) views. 89-91. Male left pedipalp: retrolateral, ventral, and prolateral views. 92. Spermathecae.

PTw 1.27-1.37, PT1 2.37-3.00, Bl 0.92-1.15; TSp 7-8, TSr 13-16.

Female (PARATyPe): Specinen preparation and condition. Female collected live in pitfall trap, preserved in same manner as holotype. Genital plate removed and stored in microvial with specimen. Color. Carapace, legs, chelicerae, dark reddish brown (5YR 3/4). Abdomen uniform reddish brown dorsally (5YR 4/3), ventrum and spinnerets pale yellow; recently collected specimens have wide dusky stripes on dorsum. Cephalothorax. Carapace 5.62 long, 4.56 wide, glabrous; generally smooth surface, pars cephalica moderately elevated. Fringe lacks setae. Foveal groove deep, moderately procurved. Eye group slightly elevated on low medial mound. AER slightly procurved, PER slightly recurved, PME, AME subequal in diameter. Sternum moderately setose, STR1 3.20, STRw 3.12. Three pairs of sternal sigilla, anterior pairs small, oval, marginal, posterior pair much larger, oval, more mesially positioned, nearly contiguous (separated by thin line of setae). Chelicerae, anterior tooth row armed with 11 teeth, posterior margin with denticle patch. Palpal endites with cuspules (ca. 37) concentrated at inner (promargin) posterior heel; labium with one cuspule, LBw 1.22, LBl 0.76. Rastellum consisting of five very stout spines positioned on mound; fringe of short spines along distal promargin extending upward from rastellum. Abdomen. Moderately setose. Legs. Leg I: 3.56, 1.80, $2.40,1.68,1.20$; leg IV: $3.60,2.00,3.12,2.60$, 1.60. Tarsus I with single staggered row of eight trichobothria. Legs I-IV with moderately light scopulae on tarsi, light scopulae on distal third of metatarsus I. PT3s $>20$, TB3s-dense patch of thin spines. Distinct preening comb on retrolateral distal surface (at tarsus-metatarsus joint) of metatarsi III, IV. Spermathecae. With relatively elongate stalk, terminating in lightly sclerotized bulb, base relatively small, heavily sclerotized (fig. 92).

MATERIAl EXAMINED: UNITED STATES: MISSISSIPPI: Calhoun Co.: $13 \mathrm{mi}$ NW Bruce 


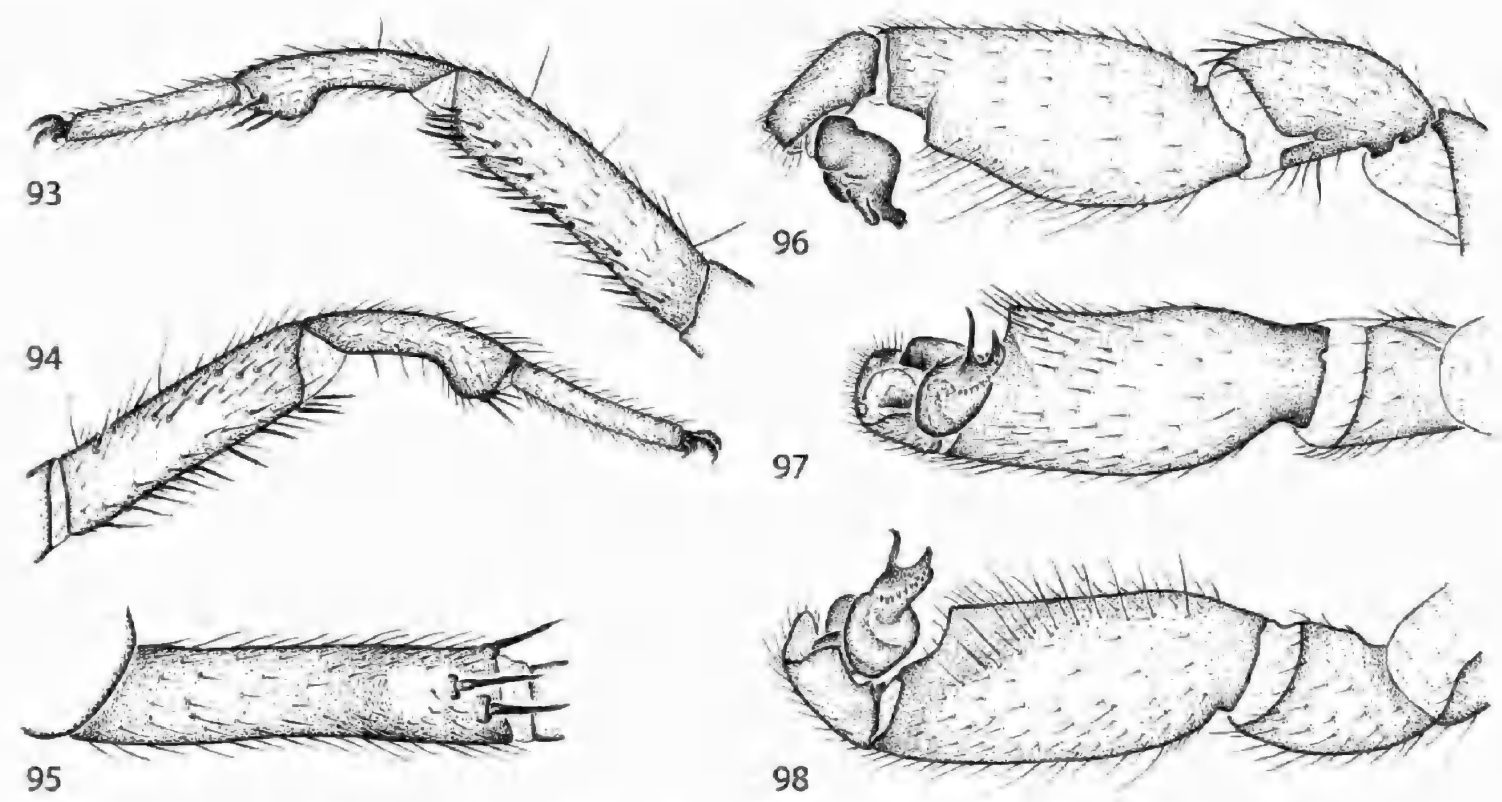

Figs. 93-98. Myrmekiaphila howelli, new species, from Newton Co., Mississippi. 93-95. Male left leg I: retrolateral, prolateral, and ventral (metarsus) víews, 96-98. Male left pedipalp: retrolateral, ventral, and prolateral views.

[34.1476 89.2557, MYR190], May 8, 1985 (P. Lago, AMNH), 1 fै. Choctaw Co.: Tombigbee National Forest, Choctaw Recreation Area, Chata Trail, $33.27277-89.14778$, elev. $192 \mathrm{~m}$ [MY2039], June 16, 2003 (B. Hendrixson, P. Marek, ECU-AMNH), 1 \% Grenada Co: T22N R3E, 31NW, Section $13[33.7295$ -90.0281, MYR185], Mar. 31-Apr. 13, 1992 (G. Snodgrass, AMNH), 48: T21N, R2E, Section 12, 13N \& R3E, Section 7S, 18N [33.6988 -90.0373, MYR186, 187], Mar. 12, 1992 (G. Snodgrass, AMNH), 60; T22N, R3E, Section 31 [33.7295 90.0281, MYR 192, 193], July 10-16, 1991, Mar, 31, 1992 (G. Baker, P. Miller, G. Snodgrass, AMNH ), 1 , 18. Lafayette Co.: $8 \mathrm{mi}$ SE Oxford, 34.6 -89.4833: [MYR172-181, 251, 294], Apr. 5 May 18, 1993 (G. Stratton, AMNH), 3 8;; [MYR296], July 8, 1992 (K. Kallies, AMNH), 1 9 . Oktibbeha Co.: Craig Springs [33.315] -88.9228, MYR247, 249, 252-254], Nov. 10 Dec. 5, 1980, Nov. 24, 1982 (G. Snodgrass, AMNH), 9 ${ }^{\imath}$. Panola Co.: $14 \mathrm{mi}$ ESE Batesville [34.1807 -89.7772, MYR 182-184], Mar. 12, 1992 (M. Caterino, AMNH), 4j. Pontotoc Co.: 1 mi SE Ecru [34.34384 -89.01433, MYR 189], May 8, 1980 (W. Cross, $\mathrm{AMNH}), 1$. Yalobusha Co: $3 \mathrm{mi}$ SE Water
Valley [34.141 -89.5876, MYR 191], May 8, 1985 (P. Lago, AMNH), 2 。.

Distribution: Known only from northern Mississippi (fig. 85).

\section{Myrmekiaphila howelli, new species}

Figures 12, 22, 85, 93-98

TrPes: Male holotype from 1 mile east of Union, [32.5716 -89.1056], Newton County, Mississippi (Feb. 12, 1983: T. Lockley), deposited in AMNH, courtesy of Dr. Patricia Miller.

ETyMology: The specific name is a patronym in honor of Dr. W. Howell of Samford University, Birmingham, Alabama.

DiAgnosis: Males can be recognized by the presence of a bifurcate palpal bulb, with the lower auxiliary prong being much shorter than the embolus, bearing a swollen, distinct accessory ledge which gives it the appearance of being doubled distally, with the tip of the prong curved, and by the ledge of the palpal tibia being short and stubby (figs. 12, 98), Females are unknown.

MAle (holotype): Specimen preparation and condition. Specimen collected live from 
"under a log", preserved in ca. 70\% ethanol. Coloration appears to be faded. Both pedipalps, leg I left side removed and stored in vial with specimen. General coloration. Carapace, chelicerae, and legs dark red (2.5YR 3/6). Abdomen very dusky red (2.5YR 2.5/2) dorsally, ventrum and spinnerets pale yellow; faint dusky stripes on dorsum. Cephalothorax. Carapace 5.94 long, 4.94 wide, glabrous, stout black bristles along fringe; surface smooth, pars cephalica elevated. Fringe, posterior margin with black bristles. Foveal groove deep, slightly procurved. Eyes on low mound. AER straight to slightly procurved, PER slightly recurved, PME, AME subequal in diameter. Sternum moderately setose, STR1 3.28, STRw 3.08. Posterior sternal sigilla large, positioned centrally, contiguous, anterior sigilla pairs small, oval, marginal, Chelicerae with distinct anterior tooth row (10 teeth), posterior margin with patch of small denticles. Palpal endites with patch of small cuspules on proximal, inner margin, labium with three cuspules, LBw 1.12, LB1 0.62 . Rastellum consisting of five very stout spines on mound. Abdomen. Setose, heavier black setae intermingled with fine black setae. Legs. Leg I: 5.12, 2.40, 4.04, 2.80, 2.56; leg IV: $5.00,2.20,4.40,4.48,2.68$. Very light tarsal scopulae present on legs I, II. Tarsus I with single, slightly staggered row of seven trichobothria. Leg I spination pattern illustrated in Figures 22, 93-95; TSp 7, TSr 11, TSrd 3. Pedipalp. Articles stout, lacking distinct spines (figs. 96-98). PTw 0.80, PTl 1.67, Bl 1.05. Ledge on distal-retrolateral surface of palpal tibia. Palpal bulb (figs. 12, 98) distally bifurcate, embolus relatively slender, auxiliary prong very thick, with swollen, distinct accessory ledge.

FEMALE: Unknown.

MATERIAL EXAMINED: Only the holotype.

Distribution: Known only from the type locality in Mississippi (fig. 85).

\section{The minuta Group}

This group includes a single species, from Florida, that may represent the sister group of all other Myrmekiaphila. Males lack the expanded ledge on the palpal tibia (fig. 102) and show only vague traces of a single embolar tooth (on the distal, rather than proximal, edge of the embolus). Alternatively, these features could represent a derived condition, thus representing a loss rather than a plesiomorphy.

\section{Myrmekiaphila minuta, new species}

Figures 13, 23, 32, 85, 99-105

TyPES: Male holotype and six male paratypes from Oldacre Woods [29.6516 -82.3248], Gainesville, Alachua Co., Florida (P. Choate; May 6, 1982), deposited in AMNH. Female paratype from Gainesville [29.6516 -82.3248]. Alachua Co., Florida (June 14, 1983; C., G. Edwards), deposited in FSCA.

Etrmology: The specific name refers to the diagnostically small size of this species.

Diagnosis: Males are easily recognized by the absence of the expanded ledge on the palpal tibia (fig. 102) and the embolus with one distal tooth, lacking subdistal serrations (figs. 13, 32). Males and females are easily distinguished from all other species on the basis of their small size $(\mathrm{CL}<5.0 \mathrm{~mm})$ and an outer carapace border that is pigmented (black/deep purple coloration).

MALE (HOLOTYPE): Specimen preparation and condition. Specimen collected live in pitfall trap, preserved in ca. 70\% ethanol. Coloration faded. Pedipalp, leg I left side removed and stored in vial with specimen. General coloration. Carapace, chelicerae, and legs yellowish red $(5 Y R \quad 4 / 6)$, outer border of carapace pigmented black/deep purple. Abdomen uniform dark reddish brown (5YR 3/2) dorsally, ventrum and spinnerets pale yellow, lacks faint stripes observed in other specimens. Cephalothorax. Carapace 4.44 long, 3.44 wide, glabrous, stout black bristles along fringe; surface smooth, pars cephalica elevated. Fringe, posterior margin with black bristles. Foveal groove deep, procurved. Eye group slightly raised, not on tubercle. AER slightly procurved, PER slightly recurved, PME, AME subequal in diameter. Sternum moderately setose, STRI 2.42, STRw 2.17. Posterior sternal sigilla large, positioned toward margin, anterior sigilla pairs small, oval, marginal. Chelicerae with distinct anterior tooth row (seven teeth), posterior margin with patch of small denticles. Palpal endites with patch of 

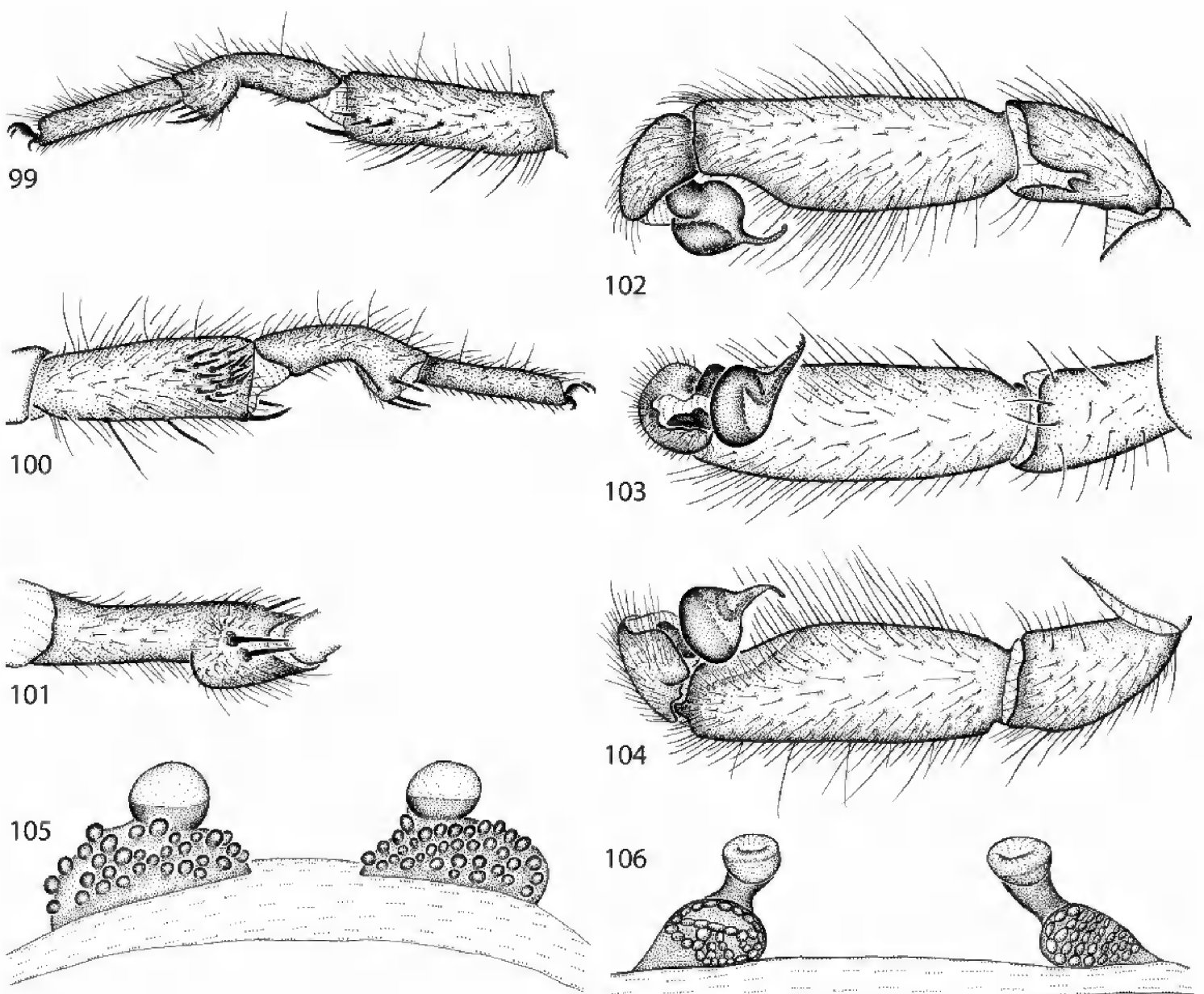

Figs. 99-106. 99-105. Myrmekiaphila minuta, new species, from Alachua Co., Florida. 99-101. Male left leg I: retrolateral, prolateral, and ventral (metarsus) views. 102-104. Male left pedipalp: retrolateral, ventral, and prolateral views. 105. Spermathecae. 106. Myrmekiaphila flavipes (Petrunkevitch) from Lawrence Co., Indiana, spermathecae.

small thin cuspules on proximal, inner margin, labium lacking cuspules, LBw 0.73, LBl 0.39. Rastellum consisting of three very stout spines on small mound. Abdomen. Setose, heavier black setae intermingled with fine black setae. Legs. Leg I: 3.28, 1.68, 2.32, 1.80, 1.68; leg IV: $3.28,1.64,2.88,2.76,1.68$. Light tarsal scopulae on legs I-IV. Tarsus I with single, staggered row of eight trichobothria. Leg I spination pattern illustrated in Figures 23, 99101; TSp 21, TSr 4, TSrd 1. Pedipalp. Articles slender, lacking distinct spines (figs. 102-104). PTw 0.70, PTl 1.90, Bl 0.82. Ledge on distalretrolateral surface of palpal tibia absent.
Palpal bulb (figs. 13, 32) not branched distally, embolus slender, lacking serrations.

Variation (3). Cl 2.82-4.44, Cw 2.25-3.44; STR1 1.78-2.17, STRw 1.50-2.42; LBw 0.490.73 , LB1 $0.24-0.39$; leg I: $2.52-3.28,1.22-$ $1.68,1.80-2.32,1.37-1.80,1.25-1.68$; leg IV: 2.37-3.28, 1.25-1.64, 2.27-2.88, 1.97-2.76, 1.20-1.68; PTw 0.39-0.70, PTl 1.53-1.90, B1 0.61-0.82; TSp 1-4, TSr 9-21.

FEMAle (PARATYPE): Specimen preparation and condition. Female collected live, wandering, preserved in ca. $70 \%$ ethanol. Genital plate removed and stored in microvial with specimens. Color. Carapace, legs, chelicerae, 
dark reddish brown (2.5YR 2.5/4), outer border of carapace pigmented black/deep purple. Abdomen uniform brown dorsally (7.5YR 4/2), ventrum and spinnerets pale yellow; specimens have wide dusky medial stripe on dorsum of abdomen. Cephalothorax. Carapace 4.40 long, 3.12 wide, glabrous; generally smooth surface, pars cephalica moderately elevated. Fringe lacks setae. Foveal groove deep, procurved. Eye group slightly elevated on low mound. AER slightly procurved, PER slightly recurved, PME, AME subequal in diameter. Sternum moderately setose, STR1 2.47, STRw 2.12. Posterior sternal sigilla large, positioned toward margins, anterior sigilla pairs small, oval, marginal. Chelicerae anterior tooth row armed with six teeth, posterior margin with denticle patch. Palpal endites with numerous cuspules (ca. 24) concentrated at inner (promargin) posterior heel; labium with two cuspules, LBw 0.83 , LB1 0.44 . Rastellum consisting of three very stout spines positioned on mound; fringe of short spines along distal promargin extending upward from rastellum. Abdomen. Moderately setose. Legs. Leg I: 2.82, 1.52, 1.80, 1.37, 1.00; leg IV: $2.50,1.75,2.37,1.82,1.00$. Tarsus I with single staggered row of seven trichobothria. Legs I-IV with moderately light scopulae on tarsi, light scopulae on distal third of metatarsus I. PT3s $>20$, TB3s-dense patch of thin spines. Distinct preening comb on retrolateral distal surface (at tarsus-metatarsus joint) of metatarsi III, IV. Spermathecae: Two simple spermathecal bulbs, in transverse row, consisting of moderately sclerotized stalk and enlarged apical bulb; base relatively large (fig. 105).

MATERIAl ExAmINed: UNITED STATES: FLORIDA: Alachua Co: no specific locality [29.7086 -82.3332, MYR171], Nov. 18, 1936 (H. Wallace, AMNH), 1 juv., [MYR298], Aug. 4, 1938 (AMNH), 1 juv.; Gainesville [29.6516 -82.3248, MYR147], June 14, 1983 (C., G., Edwards, FSCA), 1 f ; Gainesville, Oldacre Woods [MYR149], May 6, 1982 (P.

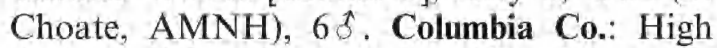
Springs, O'leno State Park, off Highway 441 [29.8544-82.6121, 052], Jan. 22, Aug. 5, 1988 (D. Corey, AMNH), $2 \hat{\sigma}$. Gadsden Co.: no specific locality [30.5633-84.6027, MYR146], July 28, 1934 (J. Kilby, AMNH), 1 juv. Leon
Co.: no specific locality [30.4946 -84.2082 , MYR300], July 30, 1950 (AMNH), 1 , 1 juv. Polk Co.: Lake Wales, Bok Tower Gardens, Route 17A [27.9359-81.5777, MYR150], Aug. 20, 1987 (D. Corey, AMNH), 28.

Distribution: Known only from central Florida and the eastern panhandle (fig. 85).

\section{UNPLACED SPECIES}

\section{Myrmekiaphila flavipes (Petrunkevitch), new combination}

Figures 1, 106

Aptostichus flavipes Petrunkevitch, 1925: 317 (female holotype from Mitchell, Lawrence Co., Indiana, in $\mathrm{AMNH}$, examined).

Diagnosis: Females can be distinguished on the basis of their unique spermathecal morphology (fig. 106): the spermathecae are widely separated and have a small base, with a relatively elongate stalk terminating in a very lightly sclerotized hemispherical bulb.

Description: Female described by Petrunkevitch (1925); males are unknown.

MATERIAL EXAMINED: UNITED STATES: INDIANA: Lawrence Co.: Mitchell [38.7328 -86.4736, MYR204], Sept. 1, 1906 (A., W. Petrunkevitch, AMNH), 1 i (holotype).

Distribution: Known only from the type locality in Mitchell, Indiana (fig. 1).

\section{ACKNOWLEDGEMENTS}

This work was supported by National Science Foundation grant DEB 0315160 to J, Bond. We thank Heather McNatt for her contributions to an early version of this manuscript (enabled through an NSF-sponsored Research Experience for Undergraduates award to the AMNH), Mohammad Shadab and Steve Thurston for their help with the illustrations and plates, Chad Spruill for help with cataloging and georeferencing specimens, and Robert Raven and Marshal Hedin for helpful comments on a draft of the manuscript. We also thank the curators and collectors who supplied specimens for their kindness (and patience!). 


\section{REFERENCES}

Atkinson, G.F. 1886, Descriptions of some new trap-door spiders; their nests and food habits. Entomologica Americana 2: 109-137.

Banks, N. 1892. Our Atypidae and Theraphosidae. Entomological News 3: 147-150.

Banks, N. 1900. Some Arachnida from Alabama. Proceedings of the Academy of Natural Sciences of Philadelphia 52: 529-543.

Bishop, S.C., and C.R. Crosby. 1926. Notes on the spiders of the southeastern United States with descriptions of new species. Journal of the Elisha Mitchell Scientific Society 41: 165-212.

Bond, J.E. 2004. The Californian euctenizine spider genus Apomastus (Araneae: Mygalomorphae: Cyrtaucheniidae); the relationship between molecular and morphological taxonomy. Invertebrate Systematics 18: 361-376.

Bond, J.E. 2005. Cyrtaucheniidae. In: D. Ubick, P. Paquin, P.E. Cushing and V. Roth (editors), Spiders of North America: an identification manual: 45-47. American Arachnological Society.

Bond, J.E., and M.C. Hedin. 2006. A total evidence assessment of the phylogeny of the diverse North American trapdoor spider subfamily Euctenizinae (Araneae, Mygalomorphae, Cyrtaucheniidae). Molecular Phylogenetics and Evolution 41: 70-85.

Bond, J.E., and B.D. Opell. 2002. Phylogeny and taxonomy of the genera of south-western North American Euctenizinae trapdoor spiders and their relatives (Araneae: Mygalomorphae; Cyrtaucheniidae). Zoological Journal of the Linnean Society of London 136: 487-534.

Cowell, R.K. 2003, Biota 2. Sunderland, MA: Sinauer Associates.
Gertsch, W.J. 1935. Spiders from the southwestern United States. American Museum Novitates 792: 1-31.

Gertsch, W.J., and H.K. Wallace. 1936. Notes on new and rare American mygalomorph spiders. American Museum Novitates 884: 1-25.

Hedin, M.C., and J.E. Bond. 2006. Molecular phylogenetics of the spider infraorder Mygalomorphae using nuclear rDNA genes (18S and 28S): conflict and agreement with the current system of classification. Molecular Phylogenetics and Evolution 41: 454 471,

Hentz, N.M. 1850. Descriptions and figures of the Araneides of the United States. Journal of the Boston Society of Natural History 6: 271-295.

Petrunkevitch, A. 1925. Descriptions of new or inadequately known American spiders. Annals of the Entomological Society of America 18: 313-322.

Petrunkevitch, A. 1929. Descriptions of new or inadequately known American spiders (second paper). Annals of the Entomological Society of America 22: 511-525.

Raven, R.J. 1985. The spider infraorder Mygalomorphae (Araneae): cladistics and systematics. Bulletin of the American Museum of Natural History 182: 1-180.

Roth, V.D. 1994. Spider genera of North America, with keys to families and genera, and a guide to literature, 3 ed. Gainesville, FL: American Arachnological Society, 203 pp.

Simon, E. 1891. Liste des espèces de la famille des Aviculariides qui habitent l'Amérique du Nord. Actes Société Linne Bordeaux 44: 307-326.

Simon, E. 1892. Histoire naturelle des araignées. Paris: Roret, 1: 1-256. 

Complete lists of all issues of the Novitates and the Bulletin are available at World Wide Web site http://library.amnh.org/pubs. Inquire about ordering printed copies via e-mail from scipubs@amnh.org or via standard mail from: American Museum of Natural History, Library-Scientific Publications, Central Park West at 79th St., New York, NY 10024. TEL: (212) 769-5545. FAX: (212) 769-5009.

This paper meets the requirements of ANSI/NISO Z39.48-1992 (Permanence of Paper). 